\title{
Cosmic evolution of the spatially resolved star formation rate and stellar mass of the CALIFA survey
}

\author{
R. López Fernández ${ }^{1}$, R. M. González Delgado ${ }^{1}$, E. Pérez ${ }^{1}$, R. García-Benito ${ }^{1}$, R. Cid Fernandes ${ }^{2}$, W. Schoenell ${ }^{3}$, \\ S. F. Sánchez ${ }^{4}$, A. Gallazzi ${ }^{5}$, P. Sánchez-Blázquez ${ }^{6}$, N. Vale Asari ${ }^{2}$, and C. J. Walcher ${ }^{7}$ \\ ${ }^{1}$ Instituto de Astrofísica de Andalucía (CSIC), PO Box 3004, 18080 Granada, Spain \\ e-mail: rosa@iaa.es \\ 2 Departamento de Física, Universidade Federal de Santa Catarina, PO Box 476, 88040-900 Florianópolis, SC, Brazil \\ ${ }^{3}$ Universidade de Sao Paulo, Instituto de Astronomía, Geofísica e Ciencias Atmosféricas, Rua do Matao 1226, \\ 05508-090 Sao Paulo, Brazil \\ ${ }^{4}$ Instituto de Astronomía, Universidad Nacional Autonóma de México, A.P. 70-264, 04510 México D.F., Mexico \\ ${ }^{5}$ INAF-Osservatorio Astrofisico di Arcetri, Largo Enrico Fermi 5, 50125 Firenze, Italy \\ ${ }^{6}$ Departamento de Física Teórica, Universidad Autónoma de Madrid, 28049 Madrid, Spain \\ ${ }^{7}$ Leibniz-Institut für Astrophysik Potsdam (AIP), An der Sternwarte 16, 14482 Potsdam, Germany
}

Received 24 November 2017 / Accepted 26 February 2018

\begin{abstract}
We investigate the cosmic evolution of the absolute and specific star formation rate (SFR, sSFR) of galaxies as derived from a spatially resolved study of the stellar populations in a set of 366 nearby galaxies from the Calar Alto Legacy Integral Field Area (CALIFA) survey. The sample spans stellar masses from $M_{\star} \sim 10^{9}$ to $10^{12} M_{\odot}$ and a wide range of Hubble types. The analysis combines images obtained with the Galaxy Evolution Explorer (GALEX; far-ultraviolet and near-ultraviolet) and Sloan Digital Sky Survey (SDSS; $u, g, r, i, z)$ with the $4000 \AA$ break, $\mathrm{H} \beta$, and [MgFe]' indices measured from the CALIFA data cubes to constrain parametric models for the star formation history (SFH), which are then used to study the cosmic evolution of the SFR density $\left(\rho_{\mathrm{SFR}}\right)$, the sSFR, the main sequence of star formation (MSSF), and the stellar mass density $\left(\rho_{\star}\right)$. Several SFH laws are used to fit the observational constrains. A delayed- $\tau$ model, SFR $\propto\left(t_{0}-t\right) \exp \left(-\left(t_{0}-t\right) / \tau\right)$, provides the best results, in good agreement with those obtained from cosmological surveys. Our main results from this model are that $(a)$ the mass currently in the inner $(\leq 0.5$ half-light radius, HLR) regions formed at earlier epochs than the mass in the outer (1-2 HLR) regions of galaxies. The time since the onset of the star formation is longer in the inner regions $\left(t_{0} \sim 13-10 \mathrm{Gyr}\right)$ than in the outer ones $\left(t_{0} \sim 11-9 \mathrm{Gyr}\right)$ for all the morphologies, while the e-folding timescale $\tau$ in the inner region is similar to or shorter than in the outer regions. These results confirm that galaxies of any Hubble type grow inside-out. (b) The sSFR declines rapidly as the Universe evolves, and faster for early- than for late-type galaxies, and for the inner than for the outer regions of galaxies. (c) The evolution of $\rho_{\mathrm{SFR}}$ and $\rho_{\star}$ agrees well with results from cosmological surveys, particularly with the recent results from the Galaxy And Mass Assembly (GAMA), the G10-Cosmological Evolution Survey (COSMOS), and the 3D Hubble Space Telescope (HST) survey. At low redshift, $z \leq 0.5$, most star formation takes place in the outer regions of late spiral galaxies, while at $z>2$, the inner regions of the progenitors of the current $\mathrm{E}$ and S0 are the main contributors to $\rho_{\mathrm{SFR}}$. $(d)$ Similarly, the inner regions of galaxies are the main contributor to $\rho_{\star}$ at $z>0.5$, growing their mass faster than the outer regions, with a lookback time at $50 \% \rho_{\star}$ of $t_{50} \sim 9$ and $6 \mathrm{Gyr}$ for the inner and outer regions. (e) The MSSF follows a power law at high redshift, with the slope evolving with time but always remaining sub-linear, in good agreement with the Illustris simulation. $(f)$ In agreement with galaxy surveys at different redshifts, the average SFH of CALIFA galaxies indicates that galaxies grow their mass mainly in a mode that is well represented by a delayed- $\tau$ model, with the peak at $z \sim 2$ and an e-folding time of $\sim 3.9$ Gyr.
\end{abstract}

Key words. galaxies: evolution - galaxies: stellar content - techniques: imaging spectroscopy

\section{Introduction}

In the past several decades, multiwavelength galaxy surveys have played a key role in the quest for understanding how galaxies form and evolve. In particular, surveys at different redshifts have been instrumental to establish that the bulk of the stellar mass $\left(M_{\star}\right)$ observed today was built up at $z \geq 2$. The most relevant observational results are that (1) the star formation rate (SFR) density in the Universe $\left(\rho_{\mathrm{SFR}}\right)$ peaks at $\sim 3.5$ Gyr after the Big Bang, at $z \sim 2$ (Lilly et al. 1996; Madau et al. 1998; Hopkins \& Beacom 2006; Fardal et al. 2007; Madau \& Dickinson 2014). (2) The stellar mass density in the Universe $\left(\rho_{\star}\right)$ evolved very little since $z \sim 1$ (Pérez-González et al. 2008; Pozzetti et al. 2010; Ilbert et al. 2013; Muzzin et al. 2013). (3) Up to $z \geq 4$, the relationbetween SFR and $M_{\star}$ is known as the main sequence of star formation (MSSF), with a logarithmic slope that is below 1 (Brinchmann et al. 2004; Noeske et al. 2007; Daddi et al. 2007; Elbaz et al. 2007; Wuyts et al. 2011; Whitaker et al. 2012; Renzini \& Peng 2015; Tasca et al. 2015; Catalán-Torrecilla et al. 2015; Cano-Díaz et al. 2016). (4) The specific star formation rate $\left(\mathrm{sSFR}=\mathrm{SFR} / M_{\star}\right)$ declines weakly with increasing galaxy mass (Salim et al. 2007; Schiminovich et al. 2007), decreases rapidly at $z<2$ (Rodighiero et al. 2010; Oliver et al. 2010; Karim et al. 2011; Elbaz et al. 2011; Speagle et al. 2014), and increases slowly at $z>2$ (Magdis et al. 2010; Stark et al. 2013; Tasca et al. 2015).

These observational results cannot be easily explained by current models of galaxy formation, which assume that galaxies grow their mass by merging of dark matter halos, progressively 
assembling more massive systems to become a single massive galaxy (Naab \& Ostriker 2016). Moreover, galaxies can grow to a large extent by fresh gas supply from the cosmic web (Kereš et al. 2005; Dekel et al. 2009a; Lilly et al. 2013). In this context, the gas accretion and SFR are expected to be associated with the specific accretion rate of dark matter (Neistein et al. 2006; Birnboim et al. 2007; Neistein \& Dekel 2008; Dutton et al. 2010). However, the specific cosmological accretion rate of baryons declines with time as $\dot{M}_{a c c} / M_{a c c} \propto(1+z)^{2.5}$ (Neistein \& Dekel 2008; Dekel et al. 2009b), while the observed sSFR $\propto(1+z)^{3}$ for galaxies at $z \leq 2$ (Oliver et al. 2010; Elbaz et al. 2011), and is constant at $z>2$ (Magdis et al. 2010; Stark et al. 2013). Some studies have shown that this particular tension can be relaxed assuming different mechanisms, such as enhanced feedback from super-winds in starbursts (Lehnert et al. 2015), or by non-trivial modifications in the semi-analytic models that involve a suppressed SFR at $z>4$ (by enhanced feedback or reduced SFR efficiency) following an initial active phase at $z>7$ (Weinmann et al. 2011).

Despite the importance of the high- $z$ work, much of our knowledge about galaxy evolution still comes from the study of nearby galaxies. Surveys at low- $z$ have been very useful to characterize the global properties of galaxies (e.g., Kauffmann et al. 2003, Blanton \& Moustakas 2009; Baldry et al. 2010), to retrieve SFR and sSFR as a function of galaxy mass (Brinchmann et al. 2004; Salim et al. 2007; Schiminovich et al. 2007), and the current value of $\rho_{\mathrm{SFR}}$ (Gallego et al. 1995; Brinchmann et al. 2004; Heavens et al. 2004). Moreover, in an alternative to surveys at different redshifts, the SFH of the Universe can be inferred by analyzing the fossil record of the current stellar populations of nearby galaxies (Panter et al. 2003, 2008; Heavens et al. 2004; Cid Fernandes et al. 2005; Ocvirk et al. 2006; Asari et al. 2007; Tojeiro et al. 2011; Koleva et al. 2011; McDermid et al. 2015; Citro et al. 2016). This method, which originally started by fitting optical colors of galaxies to study how their SFHs vary along the Hubble sequence (Tinsley 1968, 1972; Searle et al. 1973; Gallagher et al. 1984; Sandage 1986), is currently applied to fit UV-optical integrated spectra of galaxies.

More recently, integral field spectroscopy (IFS) surveys, such as the Calar Alto Legacy Integral Field Area (CALIFA; Sánchez et al. 2012, 2016a; Husemann et al. 2013; García-Benito et al. 2015), the Herschel ATLAS3D survey (Cappellari et al. 2011), the survey conducted with the Sydney-AAO Multi-object Integral field spectrograph (SAMI; Bryant et al. 2015), and the survey Mapping Nearby Galaxies at APO (MaNGA; Bundy et al. 2015; Law et al. 2015) within the Sloan Digital Sky Survey (SDSS), allow the study of the spatially resolved SFH of galaxies from spatially resolved spectroscopy (Pérez et al. 2013; Cid Fernandes et al. 2014; Sánchez-Blázquez et al. 2014a,b; González Delgado et al. 2014, 2015, 2016, 2017; Ibarra-Medel et al. 2016; Goddard et al. 2016; Sanchez et al. 2016b; de Amorim et al. 2017; García-Benito et al. 2017; Cortijo-Ferrero et al. 2017; Catalán-Torrecilla et al. 2017). In particular, with CALIFA data and using the non-parametric code STARLIGHT (Cid Fernandes et al. 2005; López Fernández et al. 2016), we have obtained the radial distribution of the current SFR and SSFR as a function of Hubble type (González Delgado et al. 2016). We have found a correlation between the stellar mass surface density $\left(\mu_{\star}\right)$ and the intensity of the star formation $\left(\Sigma_{\mathrm{SFR}}\right.$, defined as the SFR per unit area), defining a local MSSF relation with a slope similar to that of the global relation between total SFR and $M_{\star}$ (González Delgado et al. 2016; Cano-Díaz et al. 2016). This suggests that local processes are important in determining the star formation in disks. Furthermore, the radial profiles of $\Sigma_{\mathrm{SFR}}$ are very similar for all spirals. The radial profiles of the current sSFR increase outward, indicating that the recent shut-down of the star formation in spirals is progressing inside-out.

We have also studied the spatially resolved evolution of the SFR and $\Sigma_{\text {SFR }}$ as a function of galaxy mass and Hubble type (González Delgado et al. 2017). We have found that (1) galaxies form very fast independently of their current stellar mass, with the SFR peaking at $z \geq 2$. (2) At any epoch, $\Sigma_{\text {SFR }}$ scales with Hubble type. $\Sigma_{\text {SFR }}$ reaches the highest values $\left(>10^{3} M_{\odot} \mathrm{Gyr}^{-1} \mathrm{pc}^{-2}\right)$ in the central regions of current early-type galaxies (ETGs), similar to those measured in high-redshift starforming galaxies. The SFR increases sub-linearly with $M_{\star}$, such that the most massive galaxies have the highest absolute but lowest specific SFRs. (3) Evidence of an early and fast quenching is found only in the most massive $\left(M_{\star}>2 \times 10^{11} M_{\odot}\right)$ E galaxies of the sample, but not in spirals of similar mass, suggesting that halo mass is not the main mechanism for the shut-down of star formation. Less massive $\mathrm{E}$ and disk galaxies show more extended SFHs and a slower quenching than massive E galaxies.

In this paper we explore a parametric approach to "fossil cosmology", whereby colors and spectral indices predicted by analytical descriptions of the SFH are fitted to the data and used to obtain the cosmic evolution of $\rho_{\mathrm{SFR}}, \mathrm{sSFR}$, and $\rho_{\star}$. We develop a method that combines the stellar spectral indexes from CALIFA data with photometry from the Galaxy Evolution Explorer (GALEX) and the SDSS. In this way, we can harness the power of full spectral fitting (via the more relevant stellar features in the spectra of galaxies) with the spectral energy distribution (SED) by fitting the large baseline of stellar continuum provided by the UV and optical bands. This method makes the results more independent of the errors and uncertainties associated with the calibration of the galaxy spectra.

After verifying that our results are comparable to those inferred from the snapshots of galaxy evolution obtained by studies at different redshifts, we take advantage of the spatially resolved information in our data to investigate how different radial regions contribute to the evolution of $\rho_{\mathrm{SFR}}$, sSFR, and $\rho_{\star}$. This question is more easily answered with the help of fossil record studies of nearby galaxies than with redshift surveys, where spatial resolution is observationally challenging. Furthermore, since the morphologies of the galaxies in our sample are known, we can also study the role of the currently early- and late-type galaxies in the evolution of $\rho_{\mathrm{SFR}}, \mathrm{sSFR}$, and $\rho_{\star}$.

This work involves a number of assumptions and limitations with respect to studies based on snapshots at different redshifts that need to be mentioned here. In addition to limitations related to the fossil record method itself, we recall that $(a)$ the Hubble sequence has evolved with time (Delgado-Serrano et al. 2010; Cappellari 2016), so that the progenitors of, say, E and S0 in the local Universe are not necessarily also E and S0 in the past. (b) Because of stellar migrations (Roškar et al. 2008; Minchev et al. 2014), stars that are currently located at inner and outer regions in a galaxy may not be at these locations in the past. (c) The sample used in this study does not contain galaxies with strong features of interactions or mergers, but such events could well have occurred in their past histories. Our analysis cannot determine whether the mass in a galaxy has grown through mergers or smooth accretion. Mergers do play a role in galaxy evolution (Lotz et al. 2011). It is known, for instance, that the merger rate increases rapidly with redshift (Bundy et al. 2009; Bluck et al. 2009, 2012; López-Sanjuan et al. 2012, 2015; Kaviraj et al. 2015; Rodriguez-Gomez et al. 2015), possibly flattening at $z>1$ (Williams et al. 2011; Man et al. 2012; Casteels et al. 2014). 
The role played by minor or major mergers in setting the current Hubble sequence is still unknown (Lofthouse et al. 2017; Martin et al. 2017).

Notwithstanding these caveats, this study offers relevant insight on how different regions in a galaxy contribute to $\rho_{\mathrm{SFR}}(t)$, $\operatorname{sSFR}(t)$, and $\rho_{\star}(t)$, as well as how galaxy morphology relates to this cosmic evolution.

This paper is organized as follows. Section 2 describes the observations and properties of the galaxies analyzed here. In Sects. 3 and 4 we explain our method and present the results of the SFH analysis. In Sect. 5 we use these results to estimate the cosmic evolution of $\rho_{\mathrm{SFR}}, \mathrm{sSFR}$, and $\rho_{\star}$ and compare them with results obtained from surveys at different redshifts. Section 6 then examines how present-day morphology and inner and outer galaxy regions contribute to the $\rho_{\mathrm{SFR}}(t), \operatorname{sSFR}(t)$, and $\rho_{\star}(t)$ budget. The evolution of the MSSF is also discussed. Section 7 reviews our main findings. We adopt a flat cosmology for the relation between lookback time, $t(z)$, and redshift: $\Omega_{M}=$ $0.3, \Omega_{\Lambda}=0.7$, and $H_{0}=70 \mathrm{~km} \mathrm{~s}^{-1} \mathrm{Mpc}^{-1}$. The units of $M_{\star}$ are $M_{\odot}$ throughout; we do not specify them for the sake of clarity.

\section{Sample and data}

\subsection{Sample}

The main characteristics of the CALIFA mother sample are (a) an angular isophotal diameter between $45^{\prime \prime}$ and $79^{\prime \prime},(b)$ a redshift range $0.005 \leq z \leq 0.03$, and $(c)$ a color $(u-r<5)$ and magnitude $\left(-24<M_{r}<-17\right)$ covering the whole colormagnitude diagram. A full description and characterization of the mother sample is given by Walcher et al. (2014). Although the sample is not limited in volume, it can be "volume corrected", allowing us to provide estimates of the stellar mass function (Walcher et al. 2014) and other cosmological observables such as $\rho_{\mathrm{SFR}}$ (González Delgado et al. 2016). The volume-corrected distribution functions of luminosities, masses, and sizes are statistically compatible with estimates from the full SDSS when accounting for large-scale structure (see Figs. 8, 9, and 14 in Walcher et al. 2014).

The sample used in this study comprises all the galaxies from the CALIFA survey (Sánchez et al. 2012, 2016a) with COMBO cubes (the combination of the observations with the V1200 and V500 setups) in the data release 2 (García-Benito et al. 2015) for which GALEX images are available (Martin et al. 2005). This sub-sample comprises 366 galaxies and is representative of the full CALIFA mother sample, covering seven bins in morphology: E (54), S0 (48), Sa (66), Sb (58), Sbc (63), Sc (58), and Sd (19). Figure 1 shows that these proportions are very similar to those in the mother sample. In terms of stellar masses, our subsample runs from $\log M_{\star}=8.6$ to 11.9 (for a Chabrier initial mass function, IMF).

\subsection{Observations and data reduction}

The observations were carried out at the Calar Alto observatory with the $3.5 \mathrm{~m}$ telescope and the Potsdam Multi-Aperture Spectrometer PMAS (Roth et al. 2005) in the PPaK mode (Verheijen et al. 2004). PPaK is an integral field spectrograph with a field of view of $74^{\prime \prime} \times 64^{\prime \prime}$ and 382 fibers of $2.7^{\prime \prime}$ diameter each (Kelz et al. 2006). We used data calibrated with version 1.5 of the reduction pipeline, see García-Benito et al. (2015). The spectra cover the $3700-7300 \AA$ range with the same resolution as the V500 grating ( $~ 6 \AA$ of FWHM) and a spatial sampling of 1 arcsec spaxel-1.

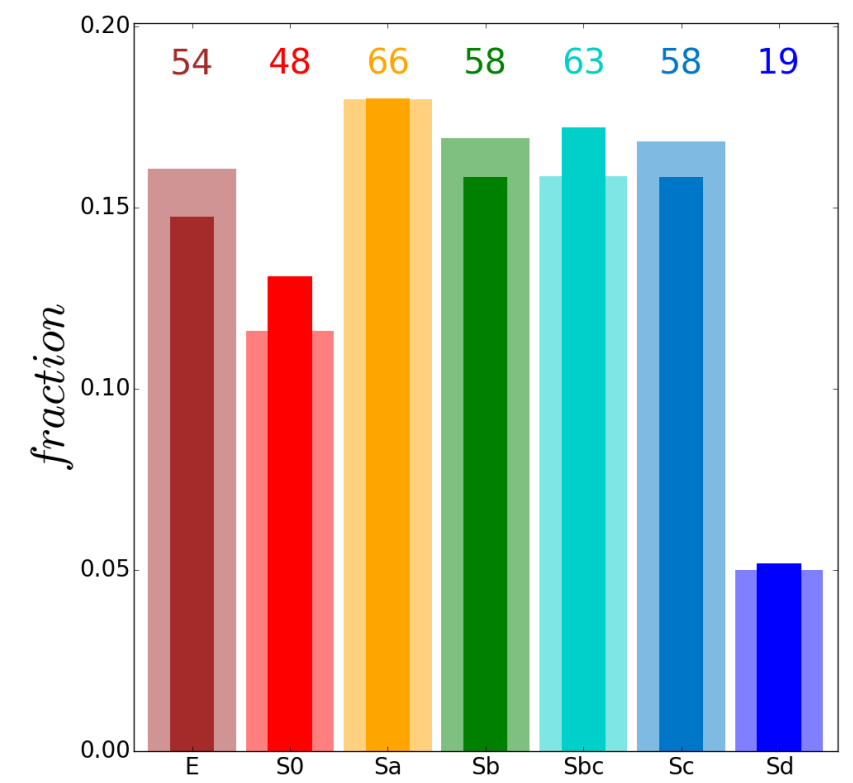

Fig. 1. Comparison of Hubble type distributions in the CALIFA mother sample (939 galaxies, broad bars) and the 366 galaxies analyzed here (narrow, darker bars). Both histograms are normalized to unit sum. The number of galaxies in each morphology bin is labeled in color.

GALEX images at far-ultraviolet (FUV; $\lambda_{\text {eff }} \sim 1542 \AA$ ) and near-ultraviolet (NUV; $\lambda_{\text {eff }} \sim 2274 \AA$ ) bands were retrieved from the GALEX website $^{1}$. We used the calibrated data products by Morrissey et al. (2007), but we performed the sky subtraction by averaging the intensity in several regions of the images. Galactic extinction was corrected for with $A_{\lambda} / E(B-V)=8.24$ and 8.20 for the FUV and NUV, respectively (Wyder et al. 2007).

Calibrated $u, g, r, I$, and $z$ images from the tenth SDSS data release (Ahn et al. 2014) were retrieved from the SDSS website $^{2}$. To correct for Galactic extinction, we used the conversion from $E(B-V)$ to total extinction $A_{\lambda}$ tabulated by Stoughton et al. (2002): $A_{\lambda} / E(B-V)=5.155,3.793,2.751,2.086$, and 1.479 for filters $u, g, r, I$, and $z$, respectively. These corrections, and those made on the FUV and NUV bands, are compatible with the Galactic extinction corrections applied to the CALIFA data cubes (García-Benito et al. 2015).

The MONTAGE software ${ }^{3}$ was used to resample the SDSS and GALEX images to the same spatial scale as CALIFA, and also to align and cut the GALEX images using World Coordinate Systems (WCS) to obtain processed FUV and NUV images with the same field of view (FoV) as our CALIFA data cubes.

Because of the inferior spatial resolution of the UV images with respect to the optical images and spectra, the spatial analysis is reduced by analyzing only three radial regions: $(i)$ between $R=0$ and 2 HLR (the whole galaxy), (ii) inside $R<0.5$ HLR (the central region, dominated by the galaxy bulge), and (iii) between $R=1$ and 2 HLR, where HLR denotes the galaxy half-light radius, measured as in Cid Fernandes et al. (2013).

The spectral indices described in Sect. 3.1 were measured from CALIFA spectra extracted within these three apertures. Fluxes in the NUV, FUV, and ugriz images were measured in the

\footnotetext{
https://asd.gsfc.nasa.gov/archive/galex

skyserver.sdss.org

3 http://montage.ipac. caltech.edu
} 
same regions, after masking foreground stars and regions with a low signal-to-noise ratio in the CALIFA data.

\section{Analysis of the star formation history}

The method for deriving the SFH for each spectrum consists of determining the best parameters of an analytical model for the SFH that simultaneously fits a combination of UV + optical photometry from GALEX and the SDSS and spectral indices from CALIFA data.

\subsection{Observational constraints and stellar population models}

In addition to the GALEX FUV and NUV photometry and the SDSS ugriz photometry, we measured the $\mathrm{H} \beta$ index, the $4000 \AA$ break $\mathrm{D}_{n} 4000$, and $[\mathrm{MgFe}]^{\prime}$ in the CALIFA spectra. We used the Lick index definition (Worthey 1994) for $\mathrm{H} \beta$ and the definition of Balogh et al. (1999) for $\mathrm{D}_{n} 4000$. These are both good indicators of the stellar population age (Bruzual \& Charlot 2003; Kauffmann et al. 2003; González Delgado et al. 2005). [MgFe $]^{\prime 4}$ is a good tracer of the stellar metallicity in a galaxy $\left(Z_{\star}\right)$. The advantage of this index with respect to other $Z_{\star}$-indicators (such as $\mathrm{Mgb}$ ) is that it is almost independent of the $\alpha / \mathrm{Fe}$ ratio (Thomas et al. 2003).

While $[\mathrm{MgFe}]^{\prime}$ and $\mathrm{D}_{n} 4000$ are measured directly in the CALIFA spectra, the $\mathrm{H} \beta$ index needs to be corrected for nebular emission. In practice, we used the synthetic value of the index obtained from a full spectral fit with STARLIGHT (Cid Fernandes et al. 2005).

To model these ten observables (seven photometric points plus three spectral indices), we used spectral models for simple stellar populations (SSP) from an updated version of the Bruzual \& Charlot (2003) models (Charlot \& Bruzual 2007, priv. comm..$^{5}$ ), where the STELIB spectral library (Le Borgne et al. 2003) is replaced by a combination of the MILES (Sánchez-Blázquez et al. 2006; Falcón-Barroso et al. 2011) and GRANADA (Martins et al. 2005) libraries. This set of models has been denoted as base $C B e$ in our previous works (González Delgado et al. 2015, 2016). The evolutionary tracks are those collectively known as Padova 1994 (Alongi et al. 1993; Bressan et al. 1993; Fagotto et al. 1994a,b; Girardi et al. 1996). The IMF is that of Chabrier (2003). The metallicity covers $\log Z_{\star} / Z_{\odot}=-2.3,-1.7,-0.7,-0.4,0$, and +0.4 , while ages run from 0 to 14 Gyr. The spectrum produced by one such SSP of initial mass $1 M_{\odot}$ is denoted by $\operatorname{SSP}_{\lambda}(t, Z)$.

These models are combined with a parametric prescription for the SFH and a simple recipe for the effects of dust to predict the observables listed above. This process is similar to that followed by Gallazzi et al. (2006) to retrieve the stellar population properties of galaxies in the SDSS survey, and, more recently, by Zibetti et al. (2017) for CALIFA data.

\subsection{Method}

\subsubsection{General aspects}

We assumed a generic $\mathrm{SFH}=\operatorname{SFH}(t ; \Theta)$, where $t$ is the lookback time and $\Theta$ is a vector of parameters including the stellar metallicity $\left(Z_{\star}\right)$, a dust attenuation parameter $\left(\tau_{V}\right)$, and parameters controlling the temporal behavior of the SFR $\psi(t)$. For instance,

\footnotetext{
4 A combination of the Lick indices Mgb, Fe5270, and Fe5335 (Thomas et al. 2003).

5 http://www . bruzual .org/ gbruzual/cb07
}

in a conventional "tau-model" $\psi(t)$ decays exponentially from an initial value $\psi_{0}$ at time $t_{0}$, with $\psi(t)=\psi_{0} \mathrm{e}^{-\left(t_{0}-t\right) / \tau}$, where $\tau$ is the SFR e-folding time. This particular model (one of the many we experimented with; see Appendix A.2) is characterized by five parameters: $\Theta=\left(\psi_{0}, t_{0}, \tau, Z_{\star}, \tau_{V}\right)$

The synthetic spectrum for a given parameterization and choice of $\Theta$ is computed with

$L_{\lambda}(\Theta)=\mathrm{e}^{-q_{\lambda} \tau_{V}} \int \operatorname{SSP}_{\lambda}(t, Z) \psi(t ; \Theta) \mathrm{d} t$,

where $q_{\lambda} \equiv \tau_{\lambda} / \tau_{V}$ denotes the attenuation law, which in our case is the one by Calzetti et al. (2000).

The goal is to explore the parameter space and constrain the parameters $\Theta$ that fit our data (indices + photometry). We used a Markov chain Monte Carlo (MCMC) method to sample the parameter space. For each sampled $\Theta$ we

- computed the predicted model spectrum (Eq. (1)) for an SFH forming a total of $1 M_{\odot}$ in stars;

- evaluated the corresponding observables;

- determined the corresponding total stellar mass formed by maximizing the likelihood of the scale-dependent observables (the photometric fluxes, in our case); and

- compared the observed and model observables, obtaining the likelihood of the data given $\Theta$.

We explain the implementation of this general method below.

\subsubsection{Bayesian inference}

Given a set of observations $O$ (seven broadband magnitudes and three spectral indices in our case), the general goal of an MCMC algorithm is to draw a set of samples $\left\{\Theta_{i}\right\}$ in the parameter space from the posterior probability density,

$p(\Theta \mid O)=\frac{p(\Theta) p(O \mid \Theta)}{p(O)}$,

where $p(\Theta)$ is the prior distribution on $\Theta$ and $p(O \mid \Theta)$ is the likelihood function. The normalization $p(O)$ is independent of $\Theta$ once we have chosen the form of the generative model, and therefore it can be omitted from the analysis.

An advantage of a Bayesian analysis is that we can marginalize over uninteresting parameters. In our case we are more interested in parameters controlling the temporal behavior of the $\mathrm{SFH}$ than in $Z_{\star}$ or $\tau_{V}$. Moreover, once the MCMC sample of $p(\Theta \mid O)$ is available, we can also obtain the expected value of any function of $\Theta$. For example, the expected value of the luminosity-weighted mean log stellar age is computed from

$\langle\log t\rangle_{L}=\sum_{\Theta_{i}} p\left(\Theta_{i} \mid O\right)\langle\log t\rangle_{L}\left(\Theta_{i}\right)$

where $\langle\log t\rangle_{L}\left(\Theta_{i}\right)$ is the mean age for a particular $\Theta_{i}$ and the sum runs over all $\Theta_{i}$ s sampled by the MCMC.

The formalism is also useful to evaluate typical uncertainties on the model parameters. For example, the uncertainties in $t_{0}$ and $\tau$ are calculated as

$$
\begin{gathered}
\left\langle t_{0}\right\rangle=\sum_{t_{0}} p\left(t_{0} \mid O\right) t_{0} ; \quad \sigma_{\left\langle t_{0}\right\rangle}^{2}=\sum_{t_{0}} p\left(t_{0} \mid O\right)\left[\left\langle t_{0}-\left\langle t_{0}\right\rangle\right]^{2}\right. \\
\langle\tau\rangle=\sum_{\tau} p(\tau \mid O) \tau ; \quad \sigma_{\langle\tau\rangle}^{2}=\sum_{\tau} p(\tau \mid O)\left[\langle\tau-\langle\tau\rangle]^{2} .\right.
\end{gathered}
$$

These estimates can then be propagated to estimate the associated uncertainties in properties such as $\rho_{\mathrm{SFR}}$ and $\rho_{\star}$, as we describe in Sects. 5.1 and 5.3. 


\subsubsection{Likelihood function}

Assuming Gaussian errors, $p(O \mid \Theta) \propto \mathrm{e}^{-\chi^{2}(\Theta) / 2}$, so that the computation of the likelihood $p(O \mid \Theta)$ reduces to the evaluation of the corresponding $\chi^{2}(\Theta)$, which in our case is split into two parts: one $\chi^{2}$ related to the $N_{\text {mag }}=7$ photometric magnitudes, and another related to the $N_{\text {Lick }}=3$ Lick indices. The latter reads

$\chi_{\text {Lick }}^{2}=\sum_{j=1}^{N_{\text {Lick }}}\left(\frac{I_{j}^{\mathrm{obs}}-I_{j}^{\mathrm{mod}}(\Theta)}{\epsilon\left(I_{j}^{\mathrm{obs}}\right)}\right)^{2}$,

where $I_{j}^{\text {obs }}$ and $\epsilon\left(I_{j}^{\text {obs }}\right)$ denote the observed index and its uncertainty, and $I_{j}^{\bmod }(\Theta)$ is the predicted value for the SFH parameters encoded in $\Theta$.

The photometric component of $\chi^{2}(\Theta)$ is

$\chi_{\mathrm{mag}}^{2}=\sum_{j=1}^{N_{\mathrm{mag}}}\left(\frac{O_{j}-M_{j}(\Theta ; 1 M \odot)-A(\Theta)}{\epsilon\left(O_{j}\right)}\right)^{2}$,

where $O_{j}$ and $\epsilon\left(O_{j}\right)$ are the observed magnitude and its error, $M_{j}\left(1 M_{\odot}\right)$ is the magnitude expected for a galaxy forming $1 M_{\odot}$ in stars, and $A=-2.5 \log M_{\star}^{\prime}$ defines the optimal ${ }^{6}$ mass formed in stars $\left(M_{\star}^{\prime}\right)$ for parameters $\Theta$.

We combine $\chi_{\text {Lick }}^{2}$ and $\chi_{\text {mag }}^{2}$ as follows:

$\chi_{\text {tot }}^{2}=\chi_{\text {mag }}^{2}+\frac{N_{\text {mag }}}{N_{\text {Lick }}} \chi_{\text {Lick }}^{2}$

where the factor $N_{\text {mag }} / N_{\text {Lick }}=7 / 3$ scales the two $\chi^{2}$ s to give the same weight to magnitudes and indices. Typically, the assumed errors in the magnitudes are $0.08,0.06,0.07,0.05,0.05,0.05$ and 0.06 mag for FUV, NUV, $u, g, r, I$, and $z$, respectively. For the indices, the average errors are $0.14 \AA, 0.09 \AA$, and $0.03 \AA$, for $\mathrm{H} \beta$, $\mathrm{D}_{n} 4000$, and $[\mathrm{MgFe}]^{\prime}$, respectively.

\section{Star formation histories as a function of Hubble type and radial region}

This section presents our results for the SFH as a function of the Hubble type and galaxy mass. We discuss the absolute and specific SFR, and the mass fraction as a function of lookback time. The spatial analysis is simplified to three radial regions: $R=0-2 \mathrm{HLR}, R<0.5 \mathrm{HLR}$, and $R=1-2 \mathrm{HLR}$, corresponding to the whole galaxy, the central region (dominated by the galaxy bulge), and outer regions (dominated by the disk). First we present our reference SFH law, the quality of the fits, and the stellar population properties obtained from the integrated spectra.

\subsection{Delayed- $\tau$ model}

We have explored a total of nine different parameterizations for $\psi(t ; \Theta)$. Appendix A.2 describes and compares these alternative models. We here restrict our discussion to the results obtained with a delayed- $\tau$ model,

$\psi(t)=k \frac{\left(t_{0}-t\right)}{\tau} \mathrm{e}^{-\left(t_{0}-t\right) / \tau}$,

where $t$ is the lookback-time, $t_{0}$ is the lookback-time onset of the star formation, $\tau$ is the SFR e-folding time, and $k$ is a normalization factor trivially related to the total mass formed in stars,

\footnotetext{
6 Optimal in the sense of $\partial \chi_{\mathrm{mag}}^{2} / \partial M_{\star}^{\prime}=0$ (see Walcher et al. 2011).
}

$M_{\star}^{\prime}=\int \psi(t) \mathrm{d} t$. Although not as popular as a simple exponential decay, this recipe has been used in several works (e.g., Bruzual \& Kron 1980; Chiosi 1980; Gavazzi et al. 2002; Lee et al. 2010; Simha et al. 2014; Chiosi et al. 2017).

This model involves a total of four parameters, $\Theta=\left(t_{0}, \tau, Z, \tau_{V}\right)$, plus the stellar mass $M_{\star}$ which is optimized as explained in the previous section. To quantify how well the model fits the data we may use the difference between the synthetic and observed quantity in units of its error: $\Delta \equiv$ (model - data)/error. The mean and one sigma values of $\Delta$ for the three spectral indices are $0.56 \pm 0.60,-0.38 \pm 1.06$, and $0.66 \pm 0.94$ for $\mathrm{D}_{n} 4000,[\mathrm{MgFe}]^{\prime}$, and $\mathrm{H} \beta$, respectively, indicating satisfactory fits. The same is true for the SDSS magnitudes, with $\Delta=0.19 \pm 1.15,-0.08 \pm 0.71,-0.46 \pm 0.29$, $-0.30 \pm 0.28$, and $0.10 \pm 0.55$ for $u, g, r, I$, and $z$, respectively. The GALEX bands are the least well fit observables, with $\Delta_{\mathrm{FUV}}=-1.38 \pm 0.81$ and $\Delta_{\mathrm{NUV}}=1.84 \pm 0.98$. As shown in Appendix, these differences between the observed and predicted UV fluxes are only reduced when a combination of two exponential functions is used for $\psi(t)$.

The quality of the fits obtained with the delayed- $\tau$ model is similar to the quality of the fits obtained with exponential or Sandage (1986) models for the SFH; in terms of $\chi^{2}$, the best is a combination of two SFH models (see Fig. A.3). Nevertheless, we favor the delayed- $\tau$ model because it results in a better match of the cosmic evolution of published $\rho_{\mathrm{SFR}}$ and sSFR from galaxy surveys.

For each galaxy, this analysis provides estimates of $t_{0}$, $\tau, Z_{\star}, \tau_{V}$, and $M_{\star}$, as well as the associated $\operatorname{SFR}(t)$ and $\operatorname{sSFR}(t)$ functions. Other properties, such as the mean stellar ages and metallicities, can be derived from them. We explore below the results of these SFH fits. The emphasis is on statistical results obtained when grouping galaxies by their Hubble type.

\subsection{Global properties as a function of Hubble type}

In Fig. 2 we present the results for the stellar mass $\left(M_{\star}\right)$, attenuation $\left(\tau_{V}\right)$, luminosity-weighted age $\left(\langle\log t\rangle_{L}\right)$, and the stellar metallicity $(\langle\log Z\rangle)$.

For this model, the metallicity is calculated as:

$\langle\log Z\rangle=\sum_{\Theta_{i}} p\left(\Theta_{i} \mid O\right) \log Z_{\star}\left(\Theta_{i}\right)$.

as a function of Hubble type. Dots represent the expected values (cf. Eq. (3)) for individual galaxies, and coloured stars mark the average for each morphology bin.

As described in our previous works, $M_{\star}$ correlates with Hubble type, with average values of $\log M_{\star}=11.18,10.98,10.81$, $10.80,10.51,9.97$, and 9.85 for E, S0, Sa, Sbc, Sc, and Sd, respectively. Extinction is not correlated with morphology, but spirals have higher $\tau_{V}$ than E and S0. $\langle\log t\rangle_{L}$ and $\langle\log Z\rangle$ scale with the Hubble type, and the ETGs are more metal rich and older than late-type spirals.

These trends with Hubble type are in good agreement with those derived by González Delgado et al. (2014, 2015) using STARLIGHT for the non-parametric spectral fits instead of the parametric models used in this paper. The agreement is also quantitative when the SSP models used for the full spectral fits are the same as were used for the parametric analysis. The differences between non-parametric and parametric properties are (mean \pm standard deviation) $0.16 \pm 0.17$ in $\log M_{\star}$, 


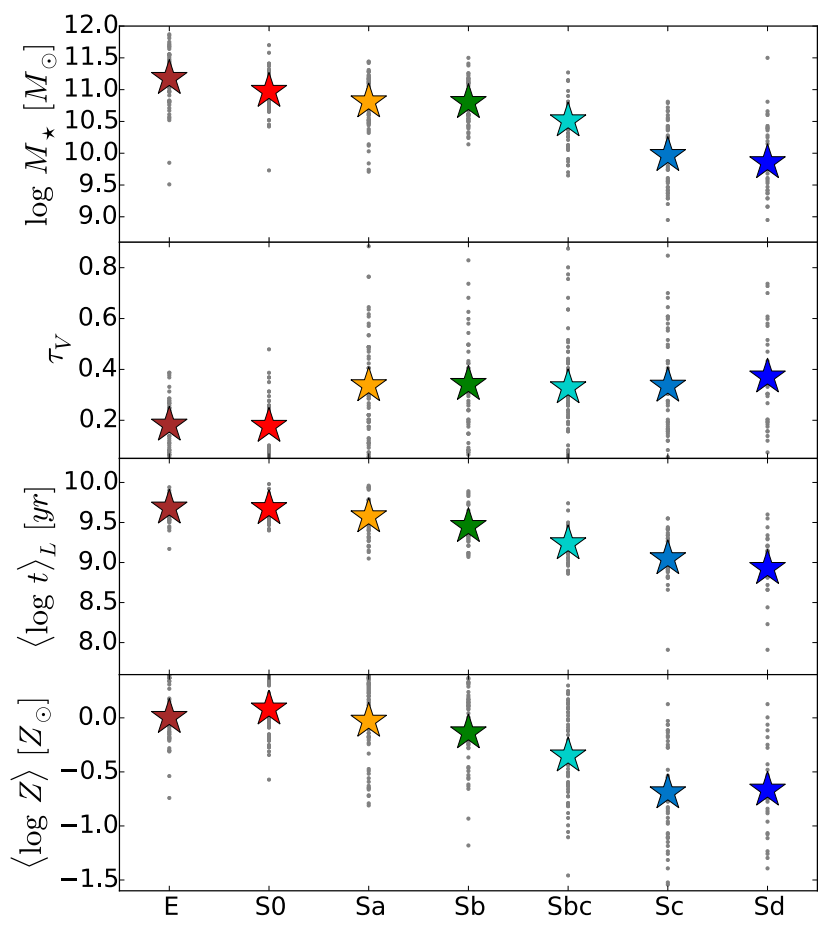

Fig. 2. Global stellar population properties as a function of the Hubble type obtained with a delayed- $\tau$ model and Chabrier IMF. From top to bottom we show the galaxy stellar mass $\left(M_{\star}\right)$, attenuation $\left(\tau_{V}\right)$, lightweighted averaged age $\left(\langle\log t\rangle_{L}\right)$, and stellar metallicity $(\langle\log Z\rangle)$. The individual galaxy values are plotted as gray dots, and the average values for each Hubble type are shown as color-coded stars.

$-0.12 \pm 0.12$ in $\tau_{V}, 0.12 \pm 0.20$ in $\langle\log t\rangle_{L}$, and $-0.07 \pm 0.37$ in $\langle\log Z\rangle$.

We note that in a few cases, our parametric fits produce unrealistically low values of $t_{0}$. For 4 of our $54 \mathrm{E}$ galaxies, for instance, the fits suggest $t_{0} \sim 4 \mathrm{Gyr}$ and low $\tau$ values. These objects are better represented by a combination of an old population with some modest recent star formation, a mixture that is not well described by a delayed- $\tau$ model. This difficulty is analogous to the one described by Trager et al. (2000), who find that single-burst models for ETGs sometimes find too young ages, and that a more reasonable scenario is one where a "frosting" of young stars is added to a old "base" population (see also Kaviraj et al. (2007)). Overall, these cases are not numerous enough to affect our main results.

\subsection{Evolution of the star formation rate}

Figure 3 shows the time evolution of the SFR for seven morphology bins obtained for the global ( $R \leq 2 \mathrm{HLR}$, left panel), central ( $R \leq 0.5 \mathrm{HLR}$, middle panel), and outer regions $(1 \leq R \leq 2$ HLR, right panel) of the galaxy. In order to make a consistent comparison with results obtained with non-parametric methods (such as those obtained with STARLIGHT, see Fig. 6 in González Delgado et al. 2017), we computed the mean SFH for each Hubble type by averaging the $\operatorname{SFR}(t)$ functions for individual galaxies in the same morphology group. The error in the mean is computed as the r.m.s. dispersion of the corresponding $\operatorname{SFR}(t)$ values divided by the square root of the number of galaxies in each bin.

These statistics are computed after excluding the 5\% of objects with smaller $t_{0}$ in each group. This minor correction was made only for cosmetic purposes. Since these outliers also have low $\tau$ values, their SFHs are concentrated in time, leading to artificial peaks in the mean $\operatorname{SFR}(t)$ curves, which are meant to be typical of a given morphology group.

Figure 3 shows that the SFRs at any epoch scale with the Hubble type, as expected because of the relations between SFR, $M_{\star}$, and morphology. E galaxies have the highest SFRs, reaching $\sim 40 M_{\odot} \mathrm{yr}^{-1}$ about 9 Gyr ago $(z \sim 1.5)$, while the lowest SFRs at the same epoch occur in Sd galaxies, with only $\sim 3 M_{\odot} \mathrm{yr}^{-1}$. We note that although the shape of $\operatorname{SFR}(t)$ for each individual galaxy follows a delayed- $\tau$ function, the mean $\operatorname{SFR}(t)$ curve does not. This happens because of the dispersion on the fitted $t_{0}$ and $\tau$ in galaxies in the same Hubble-type bin. The end result is that the mean $\operatorname{SFR}(t)$ curves shows a relatively broad plateau from $z \sim 2$ to 1 , as well as some secondary peaks. This is evident not only for E, but also for S0 and late type spirals. At recent epochs, the SFRs have declined by a factor of $\sim 40$ for ETG, and $\sim 2$ for $\mathrm{Sc}$ with respect to their values at $z \sim 1-2$.

The SFR in the inner regions (central panel) increases faster at earlier times and declines quickly after the peak, in particular for Es. At the peak of the star formation epoch (lookback time $10-12$ Gyr), the inner region contributes significantly $(\sim 55 \%)$ to the total SFR. In contrast, for the Sd galaxies, the $R<0.5$ HLR region accounts for only $16 \%$ of the total SFR.

The SFR in the outer regions (right panel) of spirals shows a behavior similar to that in the inner regions, but with a rising phase that extends to more recent times, and a declining phase that is also more extended in time. In ETGs, and in particular in Es, the peak of star formation occurs between redshift 1 and 0.5 . This result confirms our previous suggestion that $\mathrm{E}$ galaxies are actively forming or accreting stars in the outer regions between 5 and 8 Gyr ago (González Delgado et al. 2017).

\subsection{Parameters of the star formation rate}

Table 1 and Fig. 4 show the statistics (mean and standard deviation) for all values $t_{0}$ and $\tau$ as a function of Hubble type and radial region. Because the outliers (5\%; excluded from Fig. 3) are included in the statistics, the dispersion in $t_{0}$ is significant, in particular for E galaxies. The average values indicate that the onset of star formation occurs very early on: in $\mathrm{Sd}$ at $t_{0} \sim 9 \mathrm{Gyr}$, in Sc at $t_{0} \sim 10 \mathrm{Gyr}$, in Sa-Sb at $t_{0} \sim 12 \mathrm{Gyr}$, and in E and S0 at $t_{0} \sim 10.1-10.7$ Gyr. The onset of star formation in the inner regions is earlier than in the outer regions at $t_{0}(R<0.5)>t_{0}(1<R<2)$.

The timescale $\tau$ increases from $1.4 \mathrm{Gyr}$ in $\mathrm{E}$ to $4.1 \mathrm{Gyr}$ in $\mathrm{Sc}$, indicating that the period of star formation is more extended in late spirals than in ETG. The outer regions of spirals have a longer e-folding time than the inner regions, $\tau(R<0.5)<\tau(1<$ $R<2$ ); for instance, Sc galaxies typically have $\tau=5.3 \mathrm{Gyr}$ at $1<R<2$ HLR, but $3.6 \mathrm{Gyr}$ at $R<0.5$ HLR. In ETGs the inner and outer values are quite similar, with $\tau \sim 1.2$ and $1.5 \mathrm{Gyr}$, respectively.

These results confirm our earlier finding that galaxies form inside-out (Pérez et al. 2013; González Delgado et al. 2014, 2015; García-Benito et al. 2017), because the peak of star formation, at $t=t_{0}-\tau$, always occurs earlier in the inner than in the outer regions, and this inner-outer delay is very similar in all Hubble types, with $\left(t_{0}-\tau\right)_{\text {inner }}-\left(t_{0}-\tau\right)_{\text {outer }}=2.2$ Gyr on average.

\subsection{Evolution of the specific star formation rate}

The SFH of a galaxy can also be expressed through the time evolution of its sSFR, defined as the ratio between the current SFR and the stellar mass, $\operatorname{SSFR}=\operatorname{SFR}(t=0) / M_{\star}(t=0)$. This quantity provides information about the relative rate at which 

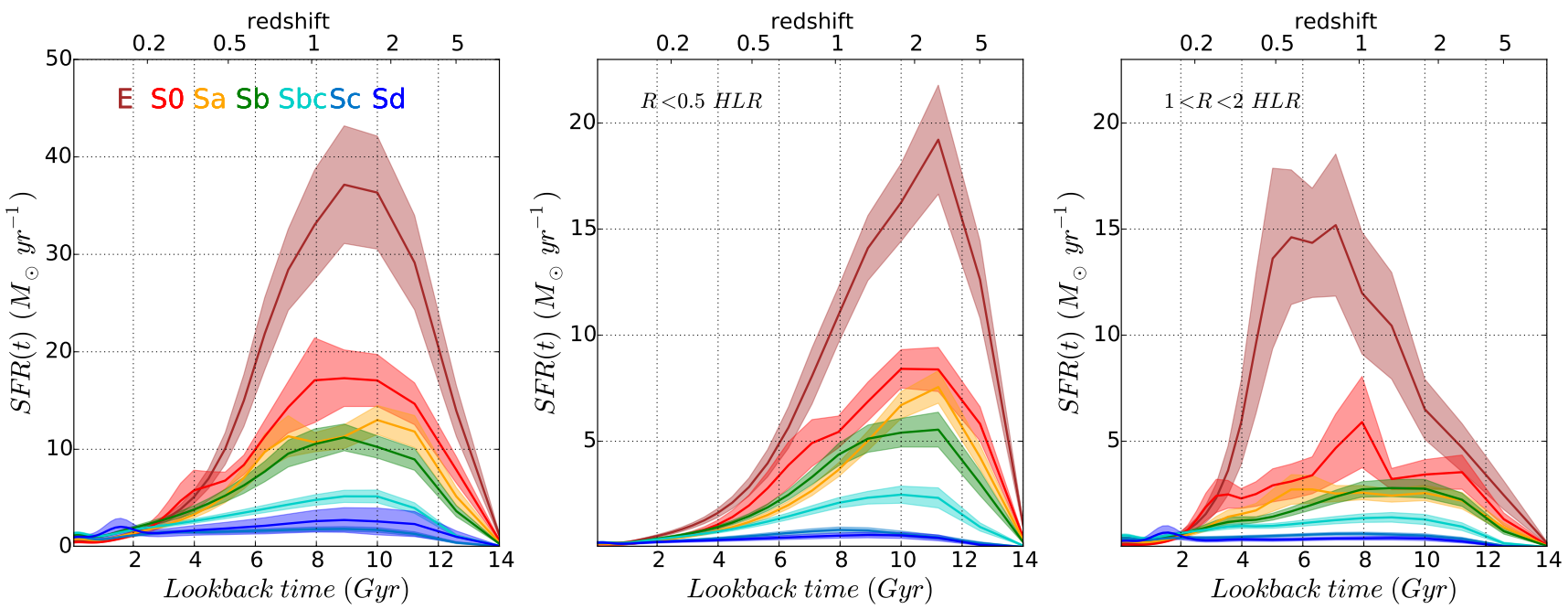

Fig. 3. Evolution of the SFR of CALIFA galaxies as a function of Hubble type; for the whole galaxy (left), the inner (middle), and outer regions (right). Solid lines are the average $\operatorname{sSFR}(t)$ of the galaxies in each morphology bin, removing only the $5 \%$ of galaxies with smallest $t_{0}$. Shaded bands around the average curves represent \pm the error in the mean.

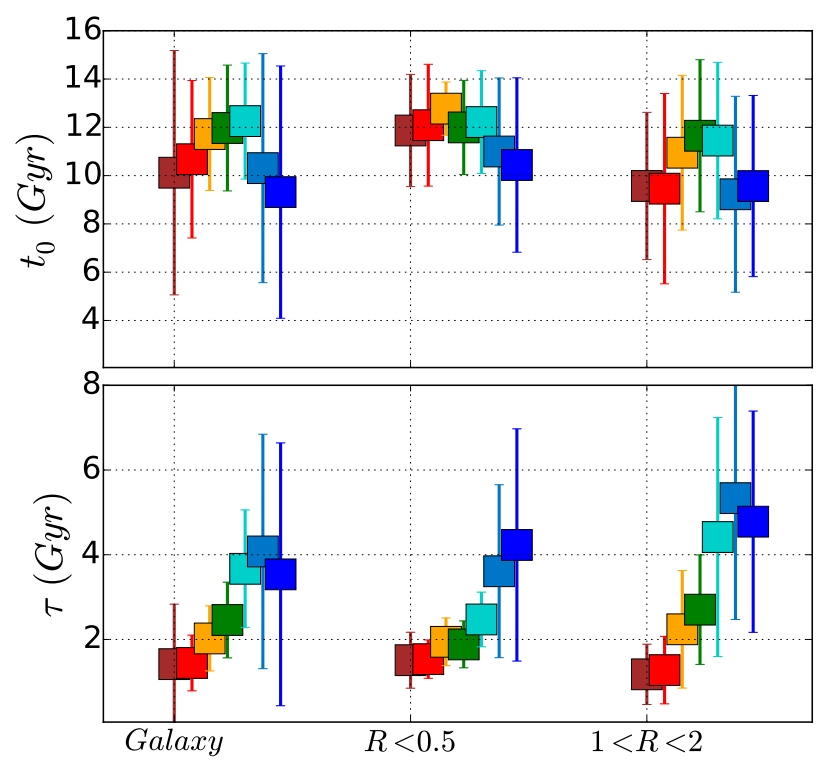

Fig. 4. Average and dispersion of the parameters $t_{0}$ and $\tau$ of a delayed$\tau$ model for the SFH, grouped into seven morphology bins. The three columns refer to results obtained for the whole galaxy (left), only the central $R<0.5$ HLR regions (middle), and $1<R<2$ HLR (right).

stars are forming now with respect to the past. The $\operatorname{sSFR}(t=0)$ declines slowly with increasing $M_{\star}$ because the MSSF is sublinear (SFR $\sim M_{\star}^{\alpha}$, with $\alpha=0.7-0.9$, Brinchmann et al. 2004; Salim et al. 2004; Renzini \& Peng 2015; Catalán-Torrecilla et al. 2015; González Delgado et al. 2016).

In previous works, using the fossil record analysis based on the full spectral fitting of CALIFA data with STARLIGHT, we were able to $(a)$ map the radial structure of the $\operatorname{sSFR}(t=0)$, and $(b)$ study temporal variations of the sSFR. Our analysis has shown that the sSFR decreases as the Universe evolves (González Delgado et al. 2017), in line with redshift surveys (Speagle et al. 2014)

One important advantage of the method proposed here based on parametric SFH is that for each galaxy we have an analytical expression for $\operatorname{SFR}(t)$ (Eq. (9)), and the values of the $\operatorname{sSFR}(t)$ are more easily derived. The $\operatorname{sSFR}(t)$ for each galaxy is calculated as
Table 1. Average and standard deviation of $t_{0}$ and $\tau$, the SFH parameters for a delayed- $\tau$ model (Eq. (9)) for seven bins in Hubble type.

\begin{tabular}{cccc}
\hline \hline M1 model & $t_{0}[\mathrm{Gyr}]$ & $\tau[\mathrm{Gyr}]$ \\
\hline & $R<0.5$ & $11.9 \pm 2.3$ & $1.5 \pm 0.7$ \\
$\mathrm{E}$ & Galaxy & $10.1 \pm 5.1$ & $1.4 \pm 1.4$ \\
& $1<R<2$ & $9.6 \pm 3.1$ & $1.2 \pm 0.7$ \\
\hline \multirow{3}{*}{$\mathrm{S} 0$} & $R<0.5$ & $12.1 \pm 2.5$ & $1.5 \pm 0.5$ \\
& galaxy & $10.7 \pm 3.3$ & $1.4 \pm 0.7$ \\
& $1<R<2$ & $9.5 \pm 3.9$ & $1.3 \pm 0.8$ \\
\hline \multirow{3}{*}{$\mathrm{Sa}$} & $R<0.5$ & $12.8 \pm 1.1$ & $1.9 \pm 0.6$ \\
& galaxy & $11.7 \pm 2.3$ & $2.0 \pm 0.8$ \\
& $1<R<2$ & $10.9 \pm 3.2$ & $2.2 \pm 1.4$ \\
\hline \multirow{3}{*}{$\mathrm{Sb}$} & $R<0.5$ & $12.0 \pm 2.0$ & $1.9 \pm 0.5$ \\
& galaxy & $12.0 \pm 2.6$ & $2.5 \pm 0.9$ \\
& $1<R<2$ & $11.6 \pm 3.1$ & $2.7 \pm 1.9$ \\
$\mathrm{~S}$ Sbc & $R<0.5$ & $12.2 \pm 2.1$ & $2.5 \pm 0.6$ \\
& galaxy & $12.3 \pm 2.4$ & $3.7 \pm 1.4$ \\
& $1<R<2$ & $11.4 \pm 3.2$ & $4.4 \pm 2.8$ \\
\hline \multirow{3}{*}{$\mathrm{Sc}$} & $R<0.5$ & $11.0 \pm 3.0$ & $3.6 \pm 2.0$ \\
& galaxy & $10.3 \pm 4.7$ & $4.1 \pm 2.8$ \\
& $1<R<2$ & $9.2 \pm 4.1$ & $5.3 \pm 2.9$ \\
\hline \multirow{2}{*}{$\mathrm{Sd}$} & $R<0.5$ & $10.4 \pm 3.6$ & $4.2 \pm 2.7$ \\
& Galaxy & $9.3 \pm 5.2$ & $3.5 \pm 3.1$ \\
& $1<R<2$ & $9.6 \pm 3.7$ & $4.8 \pm 2.6$ \\
\hline
\end{tabular}

Notes. For each type we list the results obtained for the integrated spectra, and for spectra that include regions located at $R<0.5$ HLR and $1<R<2$ HLR.

$\operatorname{SFR}(t) / M_{\star}(t)$, where the SFR at each epoch is divided by $M_{\star}(t)$ (the stellar mass of the galaxy at lookback time $t$ ), estimated as

$M_{\star}(t)=\int_{t_{0}}^{t}(1-R(t)) \operatorname{SFR}(t) \mathrm{d} t$,

where the original mass formed in stars, $\int_{t_{0}}^{t} \mathrm{SFR}(t) \mathrm{d} t$, is corrected for the mass-loss term $R(t)$. Instead of assuming a global correction, we used the prediction given by the SSP models (Bruzual \& Charlot 2003) for each population of each galaxy, based on their 

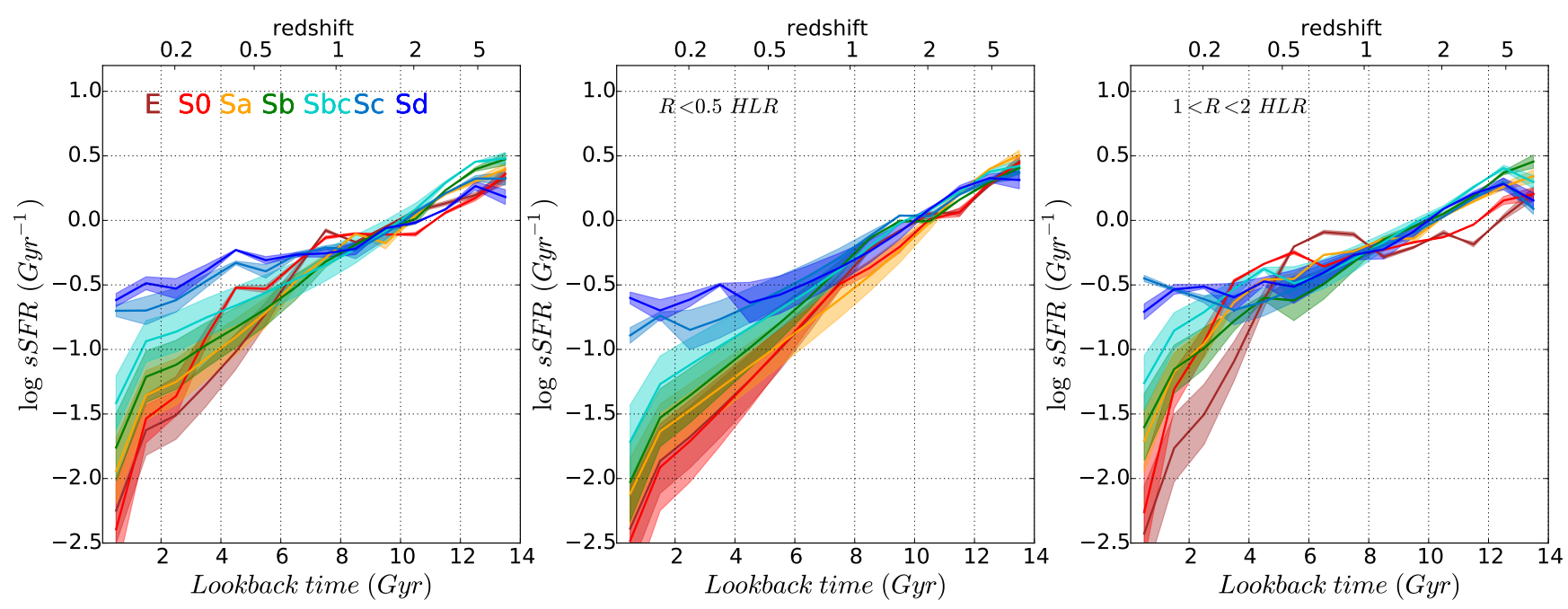

Fig. 5. Same as Fig. 3, but showing the evolution of the sSFR.

ages and metallicities. Then, the individual $\operatorname{sSFR}(t)$ was stacked as a function of Hubble type.

Figure 5 shows our results, where the full lines are average curves and shaded bands are \pm the error in the mean, that is, the dispersion divided by the square root of the number of galaxies in each class. For all morphological types the $\operatorname{sSFR}(t)$ curves decrease as the Universe evolves, but the slope is different for each Hubble type. Sd galaxies have the flattest slope, while Sa, S0, and E show steeper slopes.

The middle panel in Fig. 5 shows the $\operatorname{sSFR}(t)$ of regions located in the central 0.5 HLR. Although the behavior is similar to that of the whole galaxy, the slope is steeper, producing the shut-down of the star-formation at earlier epochs. At $z>2$, all the galaxies seem to have a common $\operatorname{sSFR}(t)$ of $\sim 2 \mathrm{Gyr}^{-1}$. In contrast, for regions currently located in the disk of spirals, the $\operatorname{sSFR}(t)$ declines more slowly (right panel in Fig. 5), indicating a longer period of star formation. The outer regions of $\mathrm{E}$ galaxies show a remarkable behavior: over the period $0.5 \leq z \leq 1$, the $\operatorname{sSFR}(t)$ runs above those of spirals, while at $z>1$ it is below. In previous works we suggested this period $0.5<z<1$ as an epoch of growth of the envelope of E and S0 through mergers.

\section{Comparison with galaxy redshift surveys}

We now set the SFHs presented in the last section into a cosmic context by applying volume corrections to obtain $\rho_{\mathrm{SFR}}(t)$, $\operatorname{sSFR}(t)$, and $\rho_{\star}(t)$. Our main goals in this section are 1) to compare our results for the cosmic evolution of the SFR, SSFR, and stellar mass obtained from the analysis of CALIFA data corresponding to the whole galaxy with the results from redshift surveys in the literature. These comparisons allow us to establish the advantages and also the limitations of the fossil record method to trace the evolution of these properties with respect to snapshots surveys of galaxy evolution obtained by studies at different redshifts. 2) We can also discuss the capability of a delayed- $\tau$ model, with respect to other parameterizations of the SFH, to trace the evolution of the SFR of the Universe.

\subsection{Cosmic star formation rate density}

One of the fundamental results obtained over the past two decades of observations from multiwavelength galaxy surveys is that the SFR density of the Universe peaked approximately 3.5 Gyr after the Big Bang, at $z \sim 2$, and declined thereafter (Lilly et al. 1996; Madau et al. 1998; Hopkins \& Beacom 2006; Fardal et al. 2007; Madau \& Dickinson 2014). Here, we extrapolate the SFR computed at each cosmic epoch from the CALIFA galaxies to derive the cosmic evolution of the SFR density $\left(\rho_{\mathrm{SFR}}\right)$ and how it breaks up into contributions from current inner and outer galaxy regions, and into the different Hubble types.

CALIFA, like many other samples, is not volume limited, but it can be volume corrected using the $V_{\max }$ method of Schmidt (1968). The volume available per galaxy, $V_{\max }$, was calculated for the CALIFA mother sample assuming that the ratio between apparent and linear isophotal size of a galaxy only depends on its angular diameter distance (Walcher et al. 2014). González Delgado et al. (2016) used this method to derive an SFR density in the local Universe $\rho_{\mathrm{SFR}}=(0.0105 \pm$ $0.0008) M_{\odot} \mathrm{yr}^{-1} \mathrm{Mpc}^{-3}$ (for a Salpeter IMF), in very good agreement with independent estimates. Furthermore, we showed that most of the current star formation occurs in the disk of spirals. Now we extend this study by calculating $\rho_{\text {SFR }}$ at different cosmic epochs using the 366 galaxies that belong to the CALIFA mother sample. We transformed our $\operatorname{SFR}(t)$ estimates into $\rho_{\mathrm{SFR}}(t)$ by adding $\operatorname{SFR}(t) / V_{\max }$ at each epoch and correcting the result by the fraction $937 / 366$ to emulate what would be obtained for the full CALIFA mother sample of 937 galaxies.

Figure 6 places our values (black stars) in the $\rho_{\mathrm{SFR}}$ vs. lookback-time (or redshift) diagram. The calculations were made at 1 Gyr time steps, and the error in each epoch was obtained by propagating the dispersion of $t_{0}$ and $\tau$ of each galaxy. $\rho_{\text {SFR }}$ shows a clear increase from $z=0$ to 1 , a plateau between $z=1$ and 3, and a decrease at higher redshift.

Figure 6 includes (gray lines) the evolution of the $\rho_{\mathrm{SFR}}$ from Madau \& Dickinson (2014), Hopkins \& Beacom (2006), and Fardal et al. (2007). It also includes the $\rho_{\mathrm{SFR}}$ from the compilation of Gunawardhana et al. $(2013,2015)$ and the results obtained by Panter et al. (2003) from the fossil record method applied to SDSS data (gray dots and squares). When necessary, the literature results were scaled to a Chabrier IMF. Our estimations are similar to the values from Fardal et al. (2007), but higher by 0.24 dex at $z \sim 0$ (see also Table 2). In contrast, our $\rho_{\mathrm{SFR}}$ at $z=0$ is in agreement with Madau \& Dickinson (2014) when their values are scaled by the change in IMF (Salpeter in 


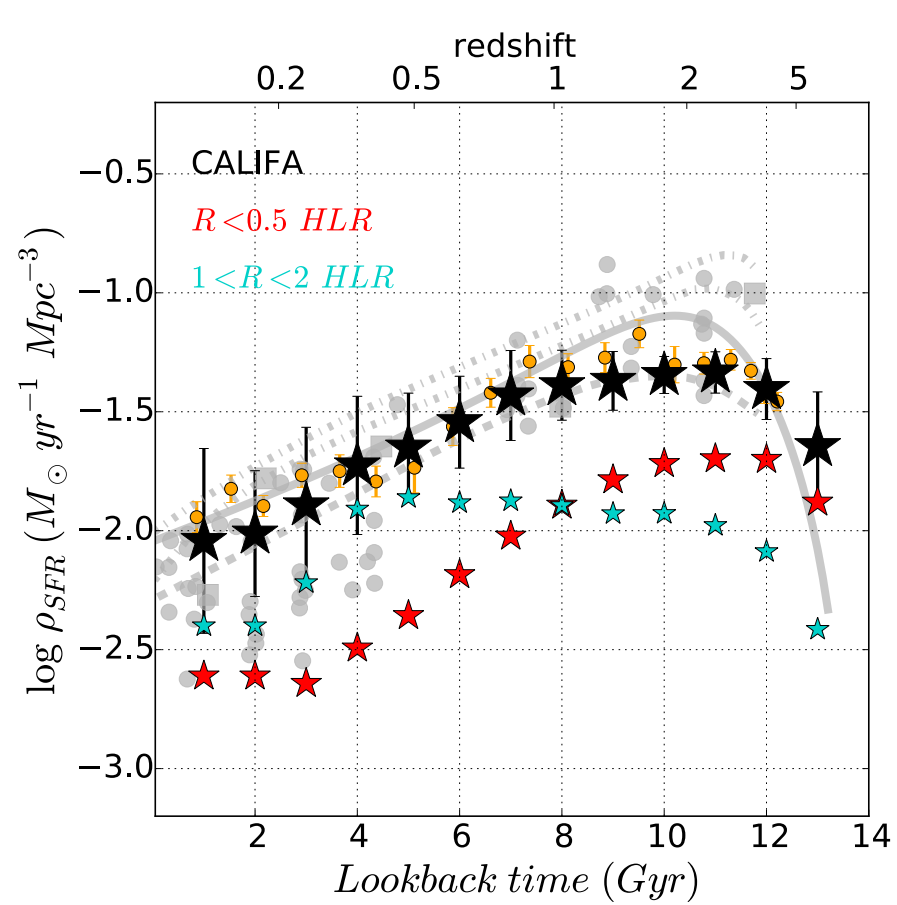

Fig. 6. Cosmic evolution of the SFR density, $\rho_{\text {SFR }}$, in this study (black stars). Blue and red stars represent the contribution to $\rho_{\mathrm{SFR}}$ of the regions between 1 and 2 HLR and within the inner 0.5 HLR, respectively. Other results are from recent determinations by Gunawardhana et al. (2013, 2015 ) and their compilation (gray points), and the redshift evolution of $\rho_{\mathrm{SFR}}$ from Hopkins \& Beacom (2006; the top two gray dotted lines are $\pm 1 \sigma$ of their relation); Madau \& Dickinson (2014; middle gray full line); Fardal et al. (2007; bottom gray dashed line); from the fossil record method applied to SDSS data by Panter et al. (2003; gray squares); and from the work of Driver et al. (2017) for data from GAMA, G10COSMOS, and 3D-HST (orange dots). When needed, literature values have been scaled to a Chabrier IMF.

Madau \& Dickinson 2014; Chabrier, in this work); but it is below by 0.24 dex at $z=2$.

Our results are also in excellent agreement with those obtained by Driver et al. (2017) using data obtained in the survey with the Galaxy And Mass Assembly (GAMA), in the G10-Cosmological Evolution Survey (COSMOS), and in the 3D Hubble Space Telescope (HST) survey (orange points in Fig. 6). The analysis of Driver and colleagues (which takes in account the effects of dust, AGN, and cosmic variance) results in a plateau in $\rho_{\mathrm{SFR}}$ between $z=3$ and 1, similar to that found in our analysis. At $z \sim 2$, our results and those from Driver et al. (2017) are a factor 1.7 below the results from Bourne et al. (2017). This latter study reports that obscured star formation dominates the total $\rho_{\mathrm{SFR}}$ in massive galaxies at all redshifts, exceeding unobscured star formation by a factor $>10$. We can think of three reasons for the discrepancy at $z \sim 2$ : (i) The sample in Bourne et al. (2017) is dominated by dust-obscured massive galaxies, and these may not be the progenitors of the CALIFA galaxies, which are representative of the current Hubble sequence. (ii) We cannot account for an extra dust obscuration at high redshift because our analysis does not assume a dust content evolution in the stellar populations. (iii) The Bourne et al. (2017) study is contaminated by AGN, which boost $\rho_{\mathrm{SFR}}$ at high redshift (as suggested by Driver et al. 2017).

On the whole, we conclude that our fossil-record estimates of $\rho_{\mathrm{SFR}}(t)$ are in good general agreement with the cosmological studies.
Table 2. $\rho_{\mathrm{SFR}}, \mathrm{sSFR}$, and $\rho_{\star}$ for redshifts $z=0,1,2$, and 5 obtained in this work.

\begin{tabular}{lccccc}
\hline \hline $\log \rho_{\mathrm{SFR}}\left[M_{\odot} \mathrm{yr}^{-1} \mathrm{Mpc}^{-3}\right]$ & $z=0$ & $z=1$ & $z=2$ & $z=5$ \\
\hline \multirow{4}{*}{ M1 } & $R<0.5$ & $-2.61 \pm 0.13$ & $-1.89 \pm 0.11$ & $-1.72 \pm 0.09$ & $-1.70 \pm 0.08$ \\
& Galaxy & $-2.04 \pm 0.38$ & $-1.39 \pm 0.14$ & $-1.34 \pm 0.07$ & $-1.41 \pm 0.12$ \\
& $1<R<2$ & $-2.40 \pm 0.11$ & $-1.89 \pm 0.31$ & $-1.93 \pm 0.14$ & $-2.09 \pm 0.25$ \\
\hline Driver et al. & -1.94 & -1.31 & -1.30 & -1.46 \\
Madau \& Dickinson & -2.05 & -1.27 & -1.10 & -1.41 \\
Fardal et al. & -2.28 & -1.42 & -1.35 & \\
\hline
\end{tabular}

$\underline{\log \mathrm{sSFR}\left[\mathrm{Gyr}^{-1}\right]}$

\begin{tabular}{lccccc}
\hline M1 & $R<0.5$ & $-1.59 \pm 0.04$ & $-0.34 \pm 0.02$ & $-0.06 \pm 0.03$ & $0.13 \pm 0.02$ \\
& Galaxy & $-1.27 \pm 0.13$ & $-0.27 \pm 0.04$ & $-0.08 \pm 0.12$ & $0.18 \pm 0.02$ \\
& $1<R<2$ & $-1.16 \pm 0.05$ & $-0.36 \pm 0.03$ & $-0.13 \pm 0.02$ & $0.08 \pm 0.02$ \\
\hline Elbaz et al. & -1.12 & -0.21 & 0.18 \\
\hline
\end{tabular}

\begin{tabular}{lccccc}
$\log \rho_{\star}\left[M_{\odot} \mathrm{Mpc}^{-3}\right]$ & & & & \\
\hline & $R<0.5$ & $7.73 \pm 0.13$ & $7.64 \pm 0.16$ & $7.46 \pm 0.19$ & $7.01 \pm 0.43$ \\
M1 & Galaxy & $8.18 \pm 0.25$ & $7.99 \pm 0.19$ & $7.78 \pm 0.24$ & $7.24 \pm 0.46$ \\
& $1<R<2$ & $7.70 \pm 0.26$ & $7.37 \pm 0.13$ & $7.10 \pm 0.18$ & $6.47 \pm 0.34$ \\
\hline Driver et al. & & 8.30 & 8.08 & 7.90 & 7.70 \\
Madau \& Dickinson & & 8.56 & 8.32 & 8.01 & 5.92 \\
\hline
\end{tabular}

\begin{tabular}{lccccc}
$\log \rho_{\star}^{\prime}\left[M_{\odot} \mathrm{Mpc}^{-3}\right]$ & & & & \\
\hline \multirow{2}{*}{ M1 } & $R<0.5$ & $8.02 \pm 0.13$ & $7.87 \pm 0.16$ & $7.66 \pm 0.19$ & $7.14 \pm 0.42$ \\
& Galaxy & $8.47 \pm 0.24$ & $8.21 \pm 0.18$ & $7.97 \pm 0.25$ & $7.35 \pm 0.44$ \\
& $1<R<2$ & $7.99 \pm 0.25$ & $7.58 \pm 0.14$ & $7.29 \pm 0.18$ & $6.60 \pm 0.34$ \\
\hline
\end{tabular}

Notes. Results from Driver et al. (2017), Madau \& Dickinson (2014), Fardal et al. (2007), and Elbaz et al. (2011) are included. The result from Driver et al. (2017) included in column $z=5$ in this table was measured at a lookback time of $12.2 \mathrm{Gyr}$.

\subsection{Cosmic specific star formation rate}

In Sect. 4.5 and Fig. 5 we have presented our results on the evolution of the $\operatorname{sSFR}(t)$ as a function of Hubble type. Here, we discuss the average sSFR at each cosmic epoch, obtained by weighting each galaxy by $w_{i}=V_{\max , i}^{-1} / \sum_{j} V_{\max , j}^{-1}$, where the sum runs over all galaxies. This volume-weighted $\operatorname{cosmic}\langle\operatorname{sSFR}(t)\rangle$ is shown as black stars in Fig. 7. The calculation for $\rho_{\mathrm{SFR}}$ was made in intervals of 1 Gyr. In Table 2 we present the results for $z=0,1$, 2 , and 5 .

As expected, $\langle\operatorname{sSFR}(t)\rangle$ decreases with cosmic time. This result is in agreement with galaxy surveys at different redshifts. To illustrate this, Fig. 7 includes the evolution of $\operatorname{sSFR}(t)$ obtained by Madau \& Dickinson (2014; gray line); after scaling to a Chabrier IMF. It also includes results (gray dots and squares) from the compilation presented by Lehnert et al. (2015) in their Fig. 2, based on measurements by Elbaz et al. (2007, 2011), Daddi et al. (2007), Dunne et al. (2009), and Rodighiero et al. (2010) in galaxy surveys at $z \leq 2$, and by Feulner et al. (2005), Stark et al. (2009, 2013), Magdis et al. (2010), and Ilbert et al. (2013) at $z \geq 2$. Figure 7 also shows (dark gray line) the best-fit relation, $\operatorname{sSFR}(t)=26 t^{2.2}$, from Elbaz et al. (2011) over the range $0 \leq z \leq 2$, equivalent to $\operatorname{SSFR}(z)=(1+z)^{3} / t_{H_{0}}$, where $t_{H_{0}}$ is the Hubble time at $z=0$. At $z>2$, the cosmological galaxy surveys show a plateau at $2 \mathrm{Gyr}^{-1}$ that differs from predictions in current galaxy-formation models (Weinmann et al. 2011). Several solutions have been proposed to bring models and observations into agreement. For example, to explain the sSFR plateau at $z>2$, Lehnert et al. (2014) have argued that at these high redshifts, the star formation must be self-regulated by high pressures that are generated by the intense star formation itself, and the increase in angular momentum with cosmic time causes a 


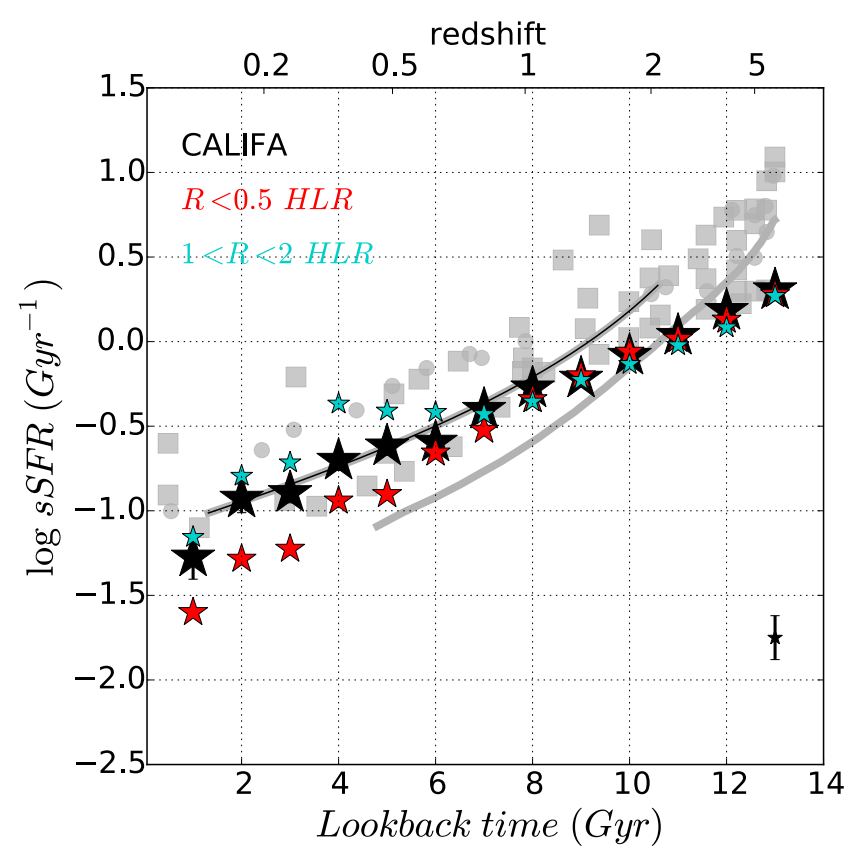

Fig. 7. Cosmic evolution of the $\operatorname{sSFR}(t)$ in the present study (black stars). The gray line is from Madau \& Dickinson (2014), the dark gray line is the $(1+z)^{3}$ relation for $z<2$ from Elbaz et al. (2007), and gray symbols are from a compilation by Lehnert et al. (2015). Red and blue stars show the $\operatorname{SSFR}(t)$ corresponding to regions at the current epoch located at $R<0.5$ HLR and $1<R<2$ HLR. The bars are the error in the mean, computed as the r.m.s. of the $\operatorname{sSFR}(t)$ values at each epoch divided by the square root of the number of galaxies in each bin.

decrease in the surface density of the accreted gas and a decrease in SSFR as the Universe evolves.

Our results are in good agreement with cosmological surveys, and they follow the time-evolution curve for $z<1$ proposed by Elbaz et al. (2011) well. Although our value at $z=0$ is $\sim 0.26$ dex below the curve of Elbaz et al. (2011), it is compatible with the lower envelope of individual values from the compilation of Lehnert et al. (2014) and above the curve of Madau \& Dickinson (2014). At $z=1$, we derive $\langle\operatorname{sSFR}(t)\rangle=0.55 \mathrm{Gyr}^{-1}$, just $10 \%$ lower than in Elbaz et al. (2011). At $1<z<2$, our values are below those from the fit estimated by Elbaz et al. (2011), but at $z>2$, our $\langle\operatorname{sSFR}(t)\rangle$ is compatible with the lower envelope of the observed galaxy surveys at $z>2$; for example, $\langle\operatorname{sSFR}(t)\rangle=0.74$ and 1.44 for $z=2$ and 5 , respectively.

\subsection{Evolution of the cosmic stellar mass density}

The stellar mass density, $\rho_{\star}$, is also a relevant observational indicator of the physical processes that regulate the mass assembly in galaxies across cosmic time. Surveys, and in particular those conducted at near-infrared wavelengths, have provided important information about the stellar mass function at redshifts $z>2$ (e.g., Dickinson et al. 2003; Fontana et al. 2003; Rudnick et al. 2003). Others that combine observations at different wavelengths were able to study the time evolution of the mass function at high and intermediate redshifts (e.g., Moustakas \& Metcalf 2003; Muzzin et al. 2013; Ilbert et al. 2013). One important conclusion from these studies was that the mass function has evolved very little since $z=1$ (e.g., Pozzetti et al. 2010).

At low-redshift, the SDSS survey also provided relevant information about the stellar mass function (e.g., Baldry et al. 2008, 2012). It has been very useful to construct a distribution

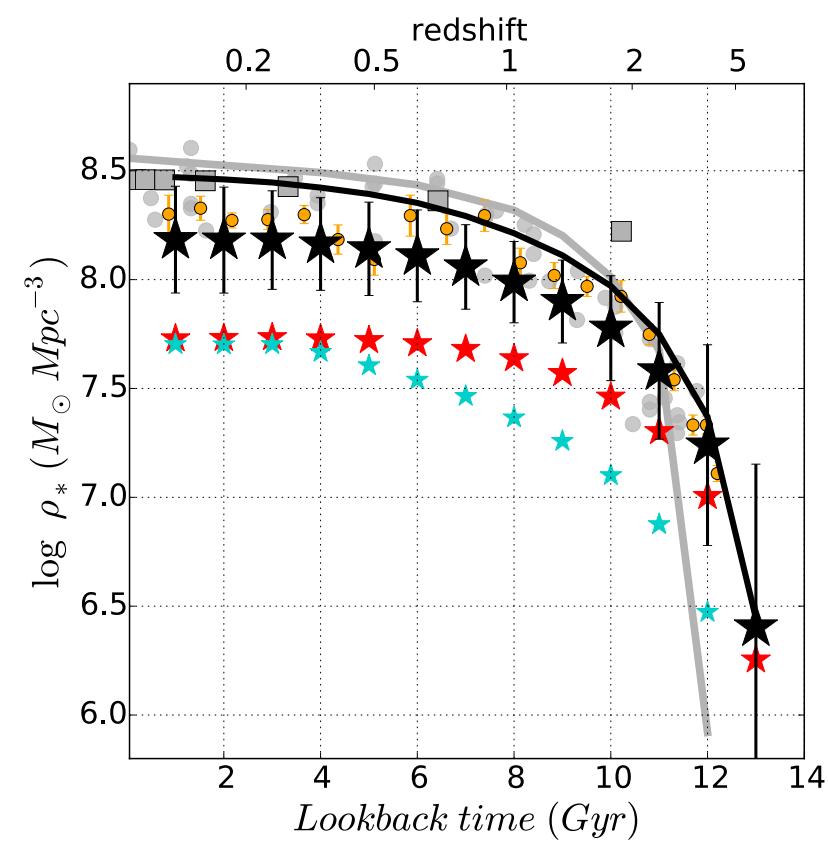

Fig. 8. Cosmic evolution of the stellar mass volume density, $\rho_{\star}$ (black stars), obtained with $M_{\star}(\mathrm{t})$ (corrected for mass loss); $\rho_{\star}^{\prime}$ (black line) obtained with $M_{\star}^{\prime}(\mathrm{t})$ (mass formed). Orange points are from the GAMA survey by Driver et al. (2017). The gray line is from Madau \& Dickinson (2014), gray squares are from Panter et al. (2007), and gray dots are from different galaxy surveys as explained in the text. Blue and red stars represent the contribution to $\rho_{\star}$ from the galaxy outer regions, $1 \leq R \leq$ 2 HLR, and inner regions, $R \leq 0.5$ HLR, respectively.

of the stellar mass as a function of age (Gallazzi et al. 2008) and to derive $\rho_{\star}$ at $z=0$, as well as to derive the cosmic evolution of $\rho_{\star}(t)$ by using the fossil record of the stellar population (Panter et al. 2007).

In our analysis, as explained in Sect. 4.5, the mass locked in stars up to a lookback time $t$ is easily obtained from the $\operatorname{SFR}(t)$ fits. The volume density $\rho_{\star}$ is then obtained from these $M_{\star}(t)$ functions just as the $\operatorname{SFR}(t)$ was used to derive $\rho_{\mathrm{SFR}}$ in Sect. 5.2. Figure 8 places our results in the $\rho_{\star}$ versus lookback time (or redshift) diagram, where the black stars and black line are the cosmic stellar mass density with and without correction for mass loss, respectively. Errors are obtained by propagating the dispersion of $t_{0}$ and $\tau$ of each galaxy. In agreement with galaxy redshift surveys, $\rho_{\star}$ shows little evolution since $z=1$, but a fast increase at higher $z$. As a reference, the $50 \%$ point is reached at $\sim 9 \mathrm{Gyr}$ lookback time.

Figure 8 also includes (gray line) the evolution of $\rho_{\star}$ from Madau \& Dickinson (2014), after scaling to a Chabrier IMF. It also includes (gray dots) $\rho_{\star}$ from the compilation of Madau \& Dickinson (2014; their Fig. 11 and Table 2); based on measurements at different redshifts by Gallazzi et al. (2008), Li \& White (2009), and Moustakas et al. (2013) for $z<1$, Arnouts et al. (2007), Pérez-González et al. (2008), Kajisawa et al. (2009), Marchesini et al. (2009), Pozzetti et al. (2010), Reddy \& Steidel (2009), Ilbert et al. (2013), and Muzzin et al. (2013) for $0.1<z<4$, Caputi et al. (2011), Yabe et al. (2009), González et al. (2011), Lee et al. (2012), and Labbé et al. (2013) for $3 \leq z \leq 5$. The results obtained by Panter et al. (2003) from the fossil record method applied to SDSS data are included as gray squares. Orange points are the recent results from GAMA/G10-COSMOS/3D-HST by Driver et al. (2017). When necessary, the literature results are scaled to a Chabrier IMF. 
We note that $\rho_{\star}$ by Madau \& Dickinson (2014) is on average $\sim 0.2$ dex higher than most of the data reported in the literature for $0<z<3$.

Comparing our results to galaxy redshift surveys, we found that a) at the highest redshifts (13 and $12 \mathrm{Gyr}$ ago), $\rho_{\star}$ is higher than in Madau \& Dickinson (2014); b) but at $z \leq 1$, our $\rho_{\star}$ is below the curve of Madau \& Dickinson (2014) and is in agreement with the lower envelope of the gray dots. Our $\rho_{\star}$ is in very good agreement with the recent results from the GAMA, G10-COSMOS, and 3D-HST multiwavelength study (Driver et al. 2017). In particular, for $z>2$, our results follow the points of Driver et al. (2017) better than the results of Madau \& Dickinson (2014) because they do not show the sudden rapid increase of the curve reported by Madau \& Dickinson (2014). Furthermore, our values for the lookback time at 12 and $13 \mathrm{Gyr}$ $\left(1.7 \times 10^{7}\right.$ and $2.5 \times 10^{6} M_{\odot} \mathrm{Mpc}^{-3}$, respectively) are also in good agreement with the results reported by Duncan et al. (2014), Grazian et al. (2015), and Song et al. (2016), which range from $2 \times 10^{7}$ to $3 \times 10^{6} M_{\odot} \mathrm{Mpc}^{-3}$.

The volume density $\rho_{\star}^{\prime}$ from $M_{\star}^{\prime}(t)$ (mass formed in stars) has a similar behavior to $\rho_{\star}$. The difference between $\rho_{\star}$ and $\rho_{\star}^{\prime}$ measures the stellar mass formed in galaxies that is returned to the interstellar medium due to stellar evolution. As expected, $\rho_{\star}^{\prime}>\rho_{\star}$ by $0.22 \mathrm{dex}(z=1)$ to $0.29 \mathrm{dex}(z=0)$; this is because for most galaxies in the sample $t_{0}>10 \mathrm{Gyr}$, and according with SSP models by Bruzual \& Charlot (2003), the stellar population needs only $\sim 4$ Gyr to lose $\sim 0.45$ of its original mass for a Chabrier IMF. Reassuringly, Driver et al. (2017) computed $\rho_{\star}^{\prime}$ by integrating $\rho_{\mathrm{SFR}}$, and comparing with $\rho_{\star}$, they obtained a returned mass fraction of $0.50 \pm 0.07$.

\subsection{SFH of galaxies: fossil cosmology versus redshift galaxy surveys}

So far, we have shown the capability of the delayed- $\tau$ model and nearby galaxies to trace the cosmic evolution of SFR and SSFR of the Universe. This SFH has been proposed to be an accurate representation of the $\mathrm{SFH}$ of galaxies on the main sequence (Speagle et al. 2014). Madau \& Dickinson (2014) have proposed a consistent picture in which the SFR density peaks at $3.5 \mathrm{Gyr}$ after the Big Bang and then declines exponentially with an e-folding time of 3.9 Gyr. Similarly to Speagle et al. (2014), Madau \& Dickinson (2014) derived their model from the fit to the evolution of $\rho_{\mathrm{SFR}}$ that was obtained from compilations of SFR using different SFR indicators and galaxy surveys, from the nearby Universe to high redshift $(z \sim 5)$.

In our case, we have derived $\operatorname{SFR}(t)$ by assuming that the SFH of nearby galaxies is well represented by a delayed- $\tau$ $\mathrm{SFH}$. This model is able to provide estimates of the cosmic SFR density and evolution of the sSFR that are compatible with $\rho_{\mathrm{SFR}}$ and sSFR derived from galaxy surveys. However, other parametric and non-parametric SFHs are able to fit the observational constraints equally well (see Appendix) and to a first approximation, provide good estimates of $\rho_{\mathrm{SFR}}$ and sSFR (e.g., González Delgado et al. 2016, 2017). Here, we discuss the similarities and differences between the different models by comparing the mass fractions, $m(t)$, obtained with different parametric and non-parametric SFH applied to the CALIFA sample and with the mass fraction derived by Madau \& Dickinson (2014).

For each galaxy, $m(t)$ is derived by dividing the stellar mass formed in each epoch by the total mass formed up to that time. González Delgado et al. (2017) previously used a non-parametric method to derive the SFHs of CALIFA galaxies and obtained

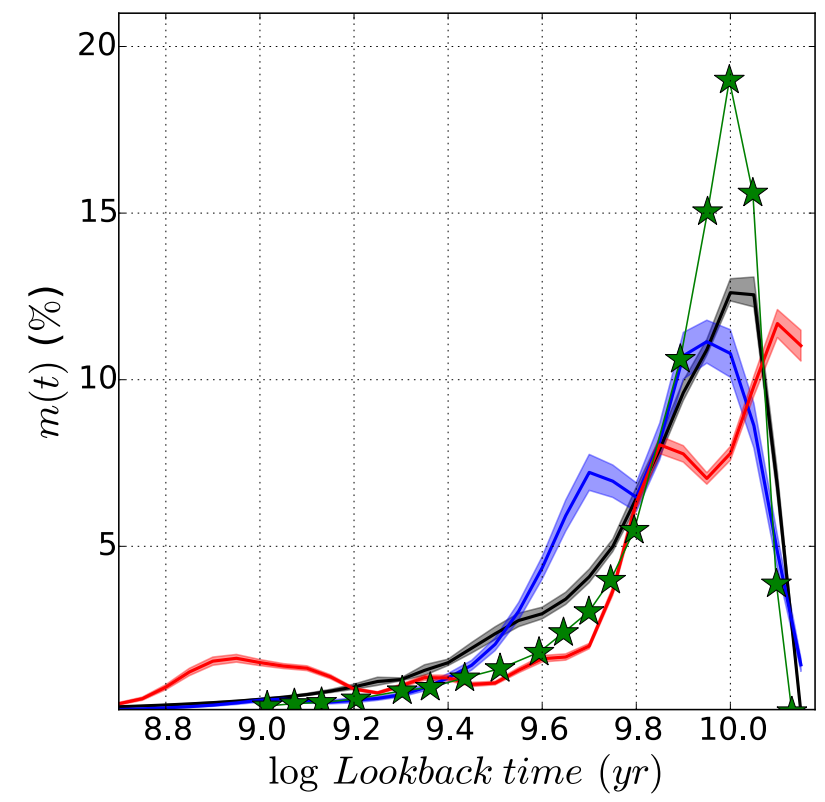

Fig. 9. Evolution of the mass fraction, $m(t)$, obtained with parametric SFHs: delayed- $\tau$ (black), a combination of two exponential SFR (blue), and STARLIGHT non-parametric (red), compared with $m(t)$ from Madau \& Dickinson (2014; green stars). The shaded bands around the mean curves approximately represent the error in the mean.

that the highest mass fractions invariably occur at the earliest times. Subsequent star formation varies systematically with $M_{\star}$, with the low $M_{\star}$ galaxies forming stars over extended periods of time, and high $M_{\star}$ galaxies exhibiting the fastest decline in $m(t)$. The behavior with morphology mimics the behavior with $M_{\star}$; for all morphologies, $m(t)$ peaks at the earliest epoch and subsequent star formation increases systematically. Here, with the parametric delayed- $\tau$ SFH, we confirm that independent of the morphology, $m(t)$ has a maximum at $z \geq 2, \sim 10 \mathrm{Gyr}$ ago, with a small shift of $\lesssim-0.1 \mathrm{dex}$ for Sc and Sd galaxies.

In order to obtain a global representation of the SFH of all the galaxies and compare with the results of Madau \& Dickinson (2014), the average $m(t)$ is obtained by weighting the mass fraction from each galaxy with $w_{i}=V_{\max , i}^{-1} / \sum_{j} V_{\max , j}^{-1}$. Figure 9 shows a comparison of our results to those of Madau \& Dickinson (2014; the histogram in their Fig. 11, and the exponential law that they proposed). From our CALIFA sample, $m(t)$ is obtained for (a) delayed- $\tau$ model, (b) a combination of two exponential SFR, and (c) using the non-parametric SFH derived from the STARLIGHT code. In addition to the description of the method used to obtain models (b) and (c), a comparison of other properties related to the mass assembly in galaxies is also given in Appendix.

The result from the delayed- $\tau$ model is quite similar to the exponential model of Madau \& Dickinson (2014), with the highest fraction peaking $3.5 \mathrm{Gyr}$ after the Big Bang, followed by a nearly exponential decline. We note that there are slightly larger fractions at lookback times between 2 and 7 Gyr ago. This behavior is somewhat different to that obtained using two exponential SFR laws; while the maximum of $m(t)$ occurred early on, at $\sim 8$ Gyr ago $(z \sim 1)$, there is a secondary epoch, $\sim 5$ Gyr ago, where the fraction of mass assembled is relevant before decaying to the actual values; this was not observed by Madau \& Dickinson (2014). For the STARLIGHT non-parametric results, $m(t)$ has two peaks 11 and 7 Gyr ago, which cover the same period of time as the peak of the delayed- $\tau$ model; then, $m(t)$ declines in a similar way as in the model of 
Madau \& Dickinson (2014). However, $m(t)$ derived from STARLIGHT shows other peaks at very recent times, $\sim 1 \mathrm{Gyr}$, which are connected to the rejuvenation epoch that late spirals experienced in the last $2 \mathrm{Gyr}$.

González Delgado et al. (2017) suggested that galaxies can grow in two different modes. For the early evolution of Sa-Sbc spirals and the entire evolution of E and SO, the logarithm of their mass fraction, $\log m(t)$, declines linearly with $\log t$, that is to say, as an exponential mode in $m(t)$ versus $\log t$. For the late-type $(\mathrm{Sc}-\mathrm{Sd})$ and low-mass galaxies, $\log m(t)$ versus $\log t$ is almost constant. We suggested that the first mode might represent the transition between the formation of a thick and a thin disk. The thick disk is a self-regulated mode, where strong outflows and turbulence drive the high intensity of the star formation rate that occurs very early on in the evolution (Lehnert et al. 2015). The second mode might be associated with the formation of the thin disk that is regulated by secular processes; a phase driven by self-gravity and energy injection from the stellar population is not relevant for global regulation (Lehnert et al. 2015).

It is interesting to note that these two modes are again reproduced here by STARLIGHT using different constraints and SSPs models. However, while the exponential mode is well derived by the parametric SFH (delayed- $\tau$ model, or the two exponential SFRs) and the exponential mode by Madau \& Dickinson (2014), the second mode for galaxy growth (not so relevant in terms of the total mass assembled for most of the galaxies) is not reproduced by the delayed- $\tau$ model. This second mode of star formation is relevant in thin disks, the main contributors to $\rho_{\mathrm{SFR}}$ and sSFR at $z=0$ (González Delgado et al. 2016). This could explain why our delayed- $\tau$ model underestimates the SSFR at lookback time $\lesssim 1$ Gyr.

\section{Discussion: cosmic evolution of the spatially resolved SFR and stellar mass}

Reassured by the satisfactory agreement between our estimates of $\rho_{\mathrm{SFR}}(t), \operatorname{sSFR}(t)$ and $\rho_{\star}(t)$ and those obtained in galaxy surveys, we now proceed and explore the spatially resolved and morphological information for our data set. The main goals of this section are to discuss 1 ) the evolution of $\rho_{\text {SFR }}, \mathrm{sSFR}$, and $\rho_{\star}$ separately for the inner and outer regions of galaxies; 2 ) we also examine the role of Hubble types in the budget of $\rho_{\mathrm{SFR}}, \mathrm{sSFR}$, and $\rho_{\star} ; 3$ ) finally, we consider the evolution of the main sequence of star formation, and the comparison of the global relation with that obtained for the inner regions of galaxies.

\subsection{Spatially resolved evolution of $\rho_{\mathrm{SFR}}, s S F R$, and $\rho_{\star}$}

Our data allow us to discuss the evolution of $\rho_{\mathrm{SFR}}, \mathrm{sSFR}$, and $\rho_{\star}$ separately for the currently inner and outer regions of galaxies. This information is interesting because spatially resolved spectroscopic observations of high-redshift galaxies are difficult to obtain, and this analysis is an attempt to infer the contributions of different galaxy sub-components, bulge and disk, to the total budget of $\rho_{\text {SFR }}$, sSFR, and $\rho_{\star}$.

To evaluate the contribution of the different galaxy regions to $\rho_{\mathrm{SFR}}$, we computed the contribution of zones located at $R<0.5$ HLR ("inner regions", mainly dominated by the current bulge component), and $1<R<2$ HLR ("outer regions", dominated by the disks). These contributions are plotted in Figs. 6-8 as red and blue points, respectively, and they are listed in Table 2 for $z=0,1,2$, and 5 . The main results are listed below.

- $\rho_{\text {SFR }}$ (Fig. 6). In the local Universe $(z \sim 0)$, the disk regions dominate $\rho_{\text {SFR }}$ (González Delgado et al. 2016), while at higher redshift the main contributors are the inner regions. At $z=0,27 \%$ of the $\rho_{\mathrm{SFR}}$ comes from the inner regions $(R<0.5$ HLR $)$, while outer regions $(1<R<2$ HLR) contribute with $44 \%$. With redshift, the central contribution increases its relevance to the total SFR density, competing with the disk-dominated regions at $z \sim 1$, where $\rho_{\mathrm{SFR}}(R<$ $0.5 \mathrm{HLR})=\rho_{\mathrm{SFR}}(1<R<2 \mathrm{HLR})$, and dominating at higher $z$. These central regions contribute $51 \%$ of $\rho_{\mathrm{SFR}}$ at $z \sim 5$.

$-\operatorname{sSFR}(t)$ (Fig. 7). At $z<1$, the outer galaxy regions have higher $\operatorname{sSFR}(t)$ than inner regions. This is because regions located in the disks are the major contributors to $\rho_{\mathrm{SFR}}$ at these redshifts. At higher $z$, regions that today are located in the inner 0.5 HLR (presumably associated with the presentday bulge) and outward of 1 HLR (today belonging to galaxy disks) have similar $\operatorname{sSFR}(t)$. These results suggests that all the regions are equally efficient in forming stars and growing their mass at high redshift.

$-\rho_{\star}$ (Fig. 8). In the local Universe $(z<0.2)$, the outer and inner regions contribute to $\rho_{\star}$ by very similar amounts. This agrees with our previous finding that the ratio of half-mass to half-light radii is close to unity (HMR/HLR $\sim 0.8$ on average; González Delgado et al. 2014). At higher redshifts the contribution from inner galaxy regions increases with respect to that from the outer zones by a factor 2.3 and 3.5 at $z=2$ and 5 (Table 2). Thus, the central regions of galaxies are the main place where $\rho_{\star}$ was built. From comparing the blue and red stars, it is clear that the central regions of galaxies have grown their mass more rapidly than the outer regions. Taking the $50 \%$ point as a reference, it was reached at lookback time 9 Gyr for the central regions and 6 Gyr ago for the outer regions. This conclusion is in agreement with our previous findings that galaxies grow their mass inside-out (Pérez et al. 2013; García-Benito et al. 2017).

\subsection{Role of morphology in the evolution of $\rho_{\mathrm{SFR}}$ and $\rho_{\star}$}

The Hubble sequence has evolved over time (Delgado-Serrano et al. 2010, e.g.). In particular, ETGs (E, S0, and Sa) can be the end product of later-type spirals transformed by mergers (e.g., Cappellari 2016 and references therein). Bearing in mind that our fossil record analysis cannot trace such morphological transformations, we now discuss the evolution of the SFR and stellar mass density as a function of the present-day morphological type.

Previously, González Delgado et al. (2016) showed that in the local Universe $(z=0), \mathrm{Sbc}, \mathrm{Sc}$, and $\mathrm{Sd}$ galaxies dominate the $\rho_{\mathrm{SFR}}$ budget. We found that galaxies of these morphologies together contribute $\sim 66 \%$ of the $\rho_{\mathrm{SFR}}$, while $\mathrm{Sa}$ and $\mathrm{Sb}$ galaxies contribute $\sim 29 \%$. Here, we discuss the contribution of current early-type (E, S0, and Sa), and late-type (LTG; Sb, Sbc, Sc, and Sd) galaxies to the evolution of $\rho_{\mathrm{SFR}}$ and $\rho_{\star}$. Tables 3 and 4 list their contributions, and Fig. 10 shows the results. A summary of the results is listed as follows:

- $\rho_{\text {SFR }}$. Present-day ETGs are the main contributors to the $\rho_{\mathrm{SFR}}$, except in the local Universe (top left panel in Fig. 10). At high redshift, the progenitors of ETGs dominate the SFR budget with $\sim 69 \%$ of the $\rho_{\mathrm{SFR}}$, while the progenitors of latetype spirals contribute with $\sim 26 \%$. However, at $z=0$, LTGs contribute with $81 \%$ of the $\rho_{\mathrm{SFR}}$, while current ETGs contribute with less than $12 \%$ to the total $\rho_{\mathrm{SFR}}$. The inner regions of the progenitors of the current ETGs contribute very little to the SFR density in the local Universe $(\sim 3 \%)$, but their contributions increase with redshift, with $21 \%$ at $z=1$ to $40 \%$ at $z=4$. However, the inner regions of LTGs contribute 
Table 3. $\rho_{\mathrm{SFR}}, \mathrm{sSFR}$, and $\rho_{\star}$ for redshifts $z=0,1,2$, and 5 obtained for early-type galaxies (E, S0, and $\mathrm{Sa}$ ).

\begin{tabular}{|c|c|c|c|c|c|}
\hline \multicolumn{2}{|c|}{$\log \rho_{\mathrm{SFR}}\left[M_{\odot} \mathrm{yr}^{-1} \mathrm{Mpc}^{-3}\right]$} & $z=0$ & $z=1$ & $z=2$ & $z=5$ \\
\hline \multirow{3}{*}{ M1 } & $R<0.5$ & $-3.51 \pm 0.09$ & $-2.07 \pm 0.10$ & $-1.86 \pm 0.09$ & $-1.81 \pm 0.06$ \\
\hline & ETG & $-2.94 \pm 0.15$ & $-1.55 \pm 0.16$ & $-1.52 \pm 0.09$ & $-1.57 \pm 0.15$ \\
\hline & $1<R<2$ & $-3.46 \pm 0.25$ & $-2.18 \pm 0.42$ & $-2.21 \pm 0.19$ & $-2.37 \pm 0.23$ \\
\hline \multicolumn{6}{|c|}{$\log$ sSFR $\left[\mathrm{Gyr}^{-1}\right]$} \\
\hline \multirow{3}{*}{ M1 } & $R<0.5$ & $-2.77 \pm 0.01$ & $-0.65 \pm 0.06$ & $-0.48 \pm 0.04$ & $-0.22 \pm 0.05$ \\
\hline & ETG & $-2.51 \pm 0.07$ & $-0.55 \pm 0.12$ & $-0.40 \pm 0.08$ & $-0.16 \pm 0.07$ \\
\hline & $1<R<2$ & $-2.38 \pm 0.02$ & $-0.64 \pm 0.05$ & $-0.49 \pm 0.06$ & $-0.35 \pm 0.06$ \\
\hline \multicolumn{6}{|c|}{$\log \rho_{\star}\left[M_{\odot} \mathrm{Mpc}^{-3}\right]$} \\
\hline \multirow{3}{*}{ M1 } & $R<0.5$ & $7.57 \pm 0.11$ & $7.51 \pm 0.13$ & $7.34 \pm 0.15$ & $6.91 \pm 0.34$ \\
\hline & ETG & $8.00 \pm 0.26$ & $7.82 \pm 0.22$ & $7.61 \pm 0.28$ & $7.08 \pm 0.51$ \\
\hline & $1<R<2$ & $7.42 \pm 0.42$ & $7.08 \pm 0.25$ & $6.81 \pm 0.34$ & $6.23 \pm 0.67$ \\
\hline
\end{tabular}

Table 4. $\rho_{\text {SFR }}$, sSFR, and $\rho_{\star}$ for redshifts $z=0,1,2$, and 5 obtained for late-type galaxies ( $\mathrm{Sb}, \mathrm{Sbc}, \mathrm{Sc}$, and $\mathrm{Sd})$.

\begin{tabular}{lccccc}
\hline \hline $\log \rho_{\mathrm{SFR}}\left[M_{\odot} y r^{-1} \mathrm{Mpc}^{-3}\right]$ & $z=0$ & $z=1$ & $z=2$ & $z=5$ \\
\hline \multicolumn{7}{c}{ M1 } & $R<0.5$ & $-2.72 \pm 0.12$ & $-2.37 \pm 0.19$ & $-2.27 \pm 0.07$ & $-2.35 \pm 0.14$ \\
& LTG & $-2.13 \pm 0.44$ & $-1.95 \pm 0.12$ & $-1.91 \pm 0.07$ & $-2.00 \pm 0.12$ \\
& $1<R<2$ & $-2.46 \pm 0.07$ & $-2.27 \pm 0.19$ & $-2.36 \pm 0.09$ & $-2.55 \pm 0.23$ \\
\hline $\log \mathrm{sSFR}\left[\mathrm{Gyr}^{-1}\right]$ & & & & \\
\hline \multirow{4}{*}{ M1 } & $R<0.5$ & $-1.66 \pm 0.08$ & $-0.64 \pm 0.04$ & $-0.27 \pm 0.06$ & $-0.13 \pm 0.06$ \\
& LTG & $-1.31 \pm 0.20$ & $-0.65 \pm 0.07$ & $-0.37 \pm 0.06$ & $-0.08 \pm 0.05$ \\
& $1<R<2$ & $-1.19 \pm 0.08$ & $-0.67 \pm 0.03$ & $-0.38 \pm 0.05$ & $-0.12 \pm 0.05$ \\
\hline $\log \rho_{\star}\left[M_{\odot} \mathrm{Mpc}^{-3}\right]$ & & & & \\
\hline \multirow{2}{*}{ M1 } & $R<0.5$ & $7.20 \pm 0.11$ & $7.05 \pm 0.16$ & $6.83 \pm 0.22$ & $6.28 \pm 0.41$ \\
& LTG & $7.65 \pm 0.21$ & $7.41 \pm 0.15$ & $7.17 \pm 0.21$ & $6.58 \pm 0.54$ \\
& $1<R<2$ & $7.29 \pm 0.42$ & $6.93 \pm 0.15$ & $6.65 \pm 0.24$ & $5.95 \pm 0.61$ \\
\hline
\end{tabular}

little to the $\rho_{\mathrm{SFR}}$, their contribution is $\sim 11 \%$ at any epoch, except in recent time, which contributes up to $21 \%$ of the total $\rho_{\mathrm{SFR}}(z=0)$. Furthermore, the outer regions of LTGs are the main contributors to the SFR density at $z=0$; at any other epoch, the outer regions of LTGs or ETGs contribute with $\leq 16 \%$ of the total budget $\rho_{\mathrm{SFR}}$.

- $\operatorname{sSFR}(t)$. Except at low redshift, the sSFR evolves similarly for ETGs and LTGs. At $z=0$, LTGs have a higher $\operatorname{sSFR}(t)$ than ETGs. This is a consequence of their greater contribution to the $\rho_{\mathrm{SFR}}$. At higher redshifts the inner regions of ETGs and LTGs evolve in similar ways, and the same holds for the outer regions of ETGs and LTGs.

$-\rho_{\star}$. At any epoch, the progenitors of present-day ETGs are the dominant population in terms of stellar mass (bottom left panel in Fig. 10). They contribute to the total budget of $\rho_{\star}$ by $69 \%$ at $z=5$ to $66 \%$ at $z=0$, but LTGs contribute with $<\sim 30 \%$ of the stellar mass density of the Universe. The inner regions of ETGs and their progenitors are also the main contributors to $\rho_{\star}$, which increases from $25 \%$ at $z=0$ to $47 \%$ at $z=5$. The inner regions of LTGs, however, contribute very little to the stellar mass density of the Universe, only $\sim 10 \%$ of the total $\rho_{\star}$ at any epoch. The outer regions of ETGs or LTGs contribute with $\leq 17 \%$ to the total budget of $\rho_{\star}$.

Thus, we can conclude that while in the local Universe the current SFR density is dominated by disks of LTGs, the SFR density at $z>1$ is dominated by the central components of present-day early-type galaxies. Moreover, the inner regions of ETGs are the main contributors to the total stellar mass density.

\subsection{Evolution of the main sequence of star formation}

Our analysis allows us to retrieve the correlation between SFR and $M_{\star}$ at different redshifts and to investigate the evolution of the slope and normalization of the MSSF. Furthermore, the effect of spatially sampling in the MSSF. Studies have shown that the correlation found in the nearby Universe (Brinchmann et al. 2004; Salim et al. 2007; Renzini \& Peng 2015; Catalán-Torrecilla et al. 2015; González Delgado et al. 2016) persists up to $z \sim 7$ (Noeske et al. 2007; Daddi et al. 2007; Pannella et al. 2009; Peng et al. 2010; Wuyts et al. 2011; Whitaker et al. 2012).

At $z=0$, it is well proved that the MSSF relation is sub-linear, although its slope varies depending on the galaxy sample selection and on the indicator used to estimate the SFR (Speagle et al. 2014). Observationally, there is also evidence that the slope and normalization of MSSF evolves with lookback time, although the role of the galaxy sample selection and the effect of extinction is not yet understood (Noeske et al. 2007; Elbaz et al. 2007; Salim et al. 2007; Schiminovich et al. 2007; Pannella et al. 2009; Whitaker et al. 2012). For example, by studying a large sample of star-forming galaxies out to $z=2.5$, Whitaker et al. (2012) found that the slope of the relation decreases at higher redshifts because the sample is more biased to dusty star-forming galaxies. Moreover, because star-forming galaxies in the past had on average a higher SFR than today (Madau \& Dickinson 2014), it is expected that the MSSF changes with time, at least in the zero-point. More recently, Speagle et al. (2014) have used a compilation of data from 25 different surveys from the literature and analyzed in a highly consistent framework the evolution of the slope and zero-point of the MSSF relation from $z=0$ to $z=6$. After accounting for the scatter between the different SFR indicators, they found that the slope and zero-point of the MSSF are likely time dependent. Thus, the slope (and zero-point) of the MSSF increases (decreases) with redshift and lookback time. In contrast, Lee et al. (2015) have found that the star-forming galaxies in the COSMOS field at $z \leq 1.3$ observed at far-infrared wavelengths follow a broken-law, $\mathrm{SFR} \propto M_{\star}^{0.88 \pm 0.06}$, below the turnover mass of $10^{10}$, and the SFR $\propto M_{\star}^{0.27 \pm 0.04}$ above $10^{10}$. Later, using data from the FourStar Galaxy Evolution Survey (ZFOURGE) at $0.5<z<4$, Tomczak et al. (2016) found that the turnover mass $\left(M_{0}\right)$ can range from $10^{9.5}$ to $10^{10.8}$, with evidence that $M_{0}$ increases with redshift.

With a completely different approach to redshift surveys, our analysis allows us to investigate the evolution of the MSSF and to compare our results with galaxy redshift surveys. Our results on the $\operatorname{SFR}(t)$ presented in Sect. 4.3 and in González Delgado et al. (2017) indeed confirm that the $\operatorname{SFR}(t)$ declines since $z \lesssim 2$, and $M_{\star}(t)$ has grown little since $z \lesssim 1$ (García-Benito et al. 2017; Pérez et al. 2013). Thus, we should expect that the MSSF at earlier epochs is shifted up with respect to the current MSSF. Furthermore, the decreasing scatter with increasing redshift can be understood because galaxies that today are out of the MSSF (because star formation has been quenched) might have been actively forming stars in the past and might have been well placed on the MSSF.

Figure 11 (upper panel) presents our MSSF extracted at four redshifts: $z=0,0.5,1$, and 2 . It also shows the relations found by fitting only current star-forming Sc galaxies, and all the galaxies of the sample. The first result to note is that while at $z=0$ the relation fitted for Sc and all the galaxies is very different, which is mainly due to ETGs that are off down the MSSF, at higher redshift, the two fits are quite similar. Considering only Sc galaxies, we obtain a slope, $a=0.66 \pm 0.17$, and a zeropoint, $b=-6.55 \pm 0.09$, at $z=0$, and $a=0.91 \pm 0.04$ and $b=-8.20 \pm 0.03$ at $z=2$. If all the galaxies are included, the slope (zero-point) also increases (decreases) significantly with redshift (see Table 5). 

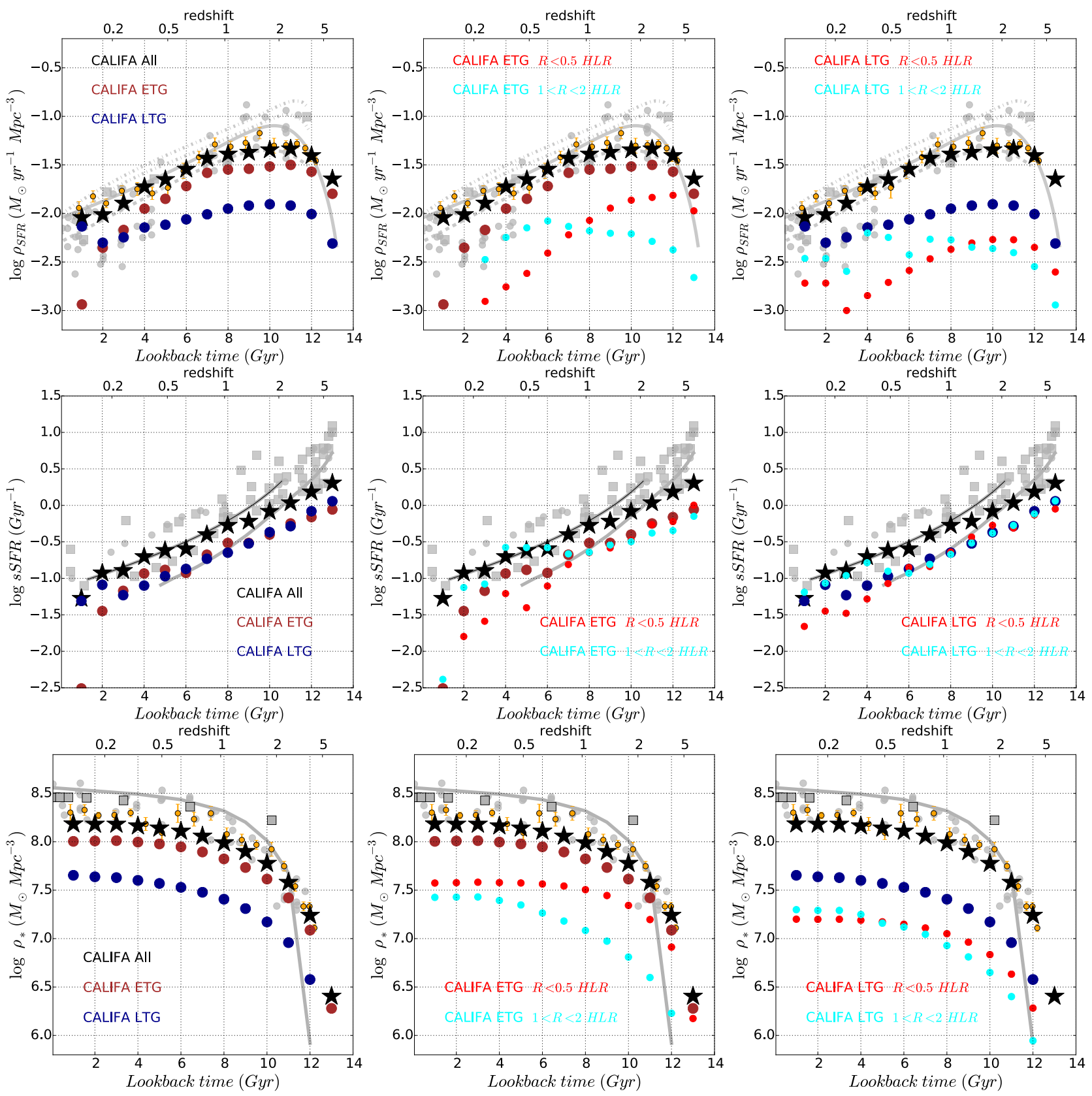

Fig. 10. Cosmic evolution of the SFR density, $\rho_{\mathrm{SFR}}$ (upper panels), the sSFR(t) (middle panels), and the stellar mass volume density, $\rho_{\star}($ lower panels) as a function of the morphology: early-type (E, S0, and Sa) and late-type ( $\mathrm{Sb}, \mathrm{Sbc}, \mathrm{Sc}$, and Sd) galaxies. The contribution of the inner $(R<0.5 \mathrm{HLR})$ and outer $(1<R<2 \mathrm{HLR})$ regions of ETGs and LTGs are listed in the central and right panels, respectively.

Two more interesting results are first that the scatter in the MSSF decreases significantly with redshift. The progenitors of all the galaxies in the sample were in the MSSF at $z \geq 2$. Second, the progenitors of E and S0 were in or above the MSSF at $z=1$.

Our results are in agreement with those of Speagle et al. (2014) and Chiosi et al. (2017), in the sense that the MSSF slope is time dependent and flattens from high to low redshift. However, this slope does not decrease linearly with the age of the Universe because our data show relatively little change from $z=2$ to $z=1$. The flattening from $z=1$ to 0 , however, is equal to their prediction.

Figure 11 also shows (purple line) the results for the MSSF by the Illustris cosmological hydrodynamical simulation of galaxy formation (Nelson et al. 2015) and observational results from a compilation by Behroozi et al. (2013) that we took from
Fig. 1 in Sparre et al. (2015). The results for $z=0.5$ are taken from the Illustris simulation at $0.5<z<1$ as presented in Fig. 6 in Tomczak et al. (2016). Figure 11 shows that the Illustris simulation at $z=1$ and 2 follows our results very well, and the trend of galaxies going out from the main sequence at $z \leq 0.5$ also agrees well. At any redshift, the points from the compilation by Behroozi et al. (2013) have a flatter slope than our results and the Illustris simulation, and at $z=1,2$, they are above the MSSF traced by Illustris and our MSSF fit. However, these points are located in a region populated by the progenitors of current ETGs (E, S0, and Sa) that were above the MSSF at $z=1,2$.

Other high-redshift galaxy surveys have also shown that passive galaxies do exist up to at least $z \sim 2$, in particular at high masses (e.g., Cimatti et al. 2004; Glazebrook et al. 2004), and that the red sequence of galaxies was present at $z \approx 1$ 

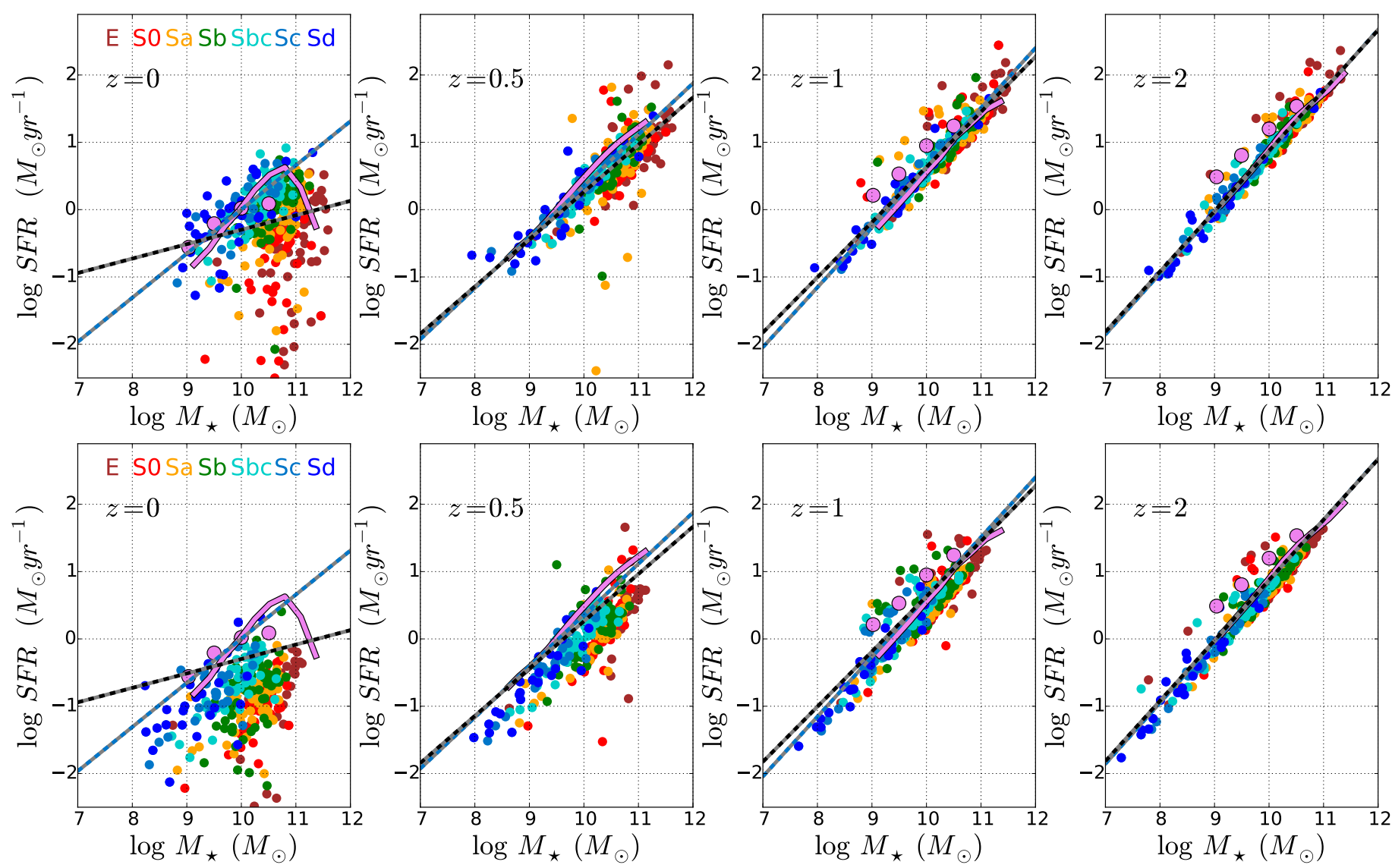

Fig. 11. Upper panels: evolution of MSSF from $z=2$ (right panel) to $\mathrm{z}=0$ (left panel). Galaxies are color-coded according to morphology. Lower panels: same as in the upper panels, but using only the contributions to SFR and $M_{\star}$ of the present-day galaxy inner regions $(R \leq 0.5$ HLR). Blue-gray and black-gray lines (in upper and lower panels) are fits to $\log \mathrm{SFR}-\log M_{\star}$ for Sc and for all the galaxies in this study. Large purple dots are from the compilation by Behroozi et al. (2013), and the purple line is the MSSF from the Illustris simulation by Sparre et al. (2015) and Tomczak et al. (2016).

Table 5. Parameters of $\log \operatorname{SFR}\left(M_{\odot} \mathrm{yr}^{-1}\right)=\mathrm{a} \log M_{\star}\left(\mathrm{M}_{\odot}\right)+\mathrm{b}$ fits of the MSSF at different redshifts obtained for Sc and all the galaxies.

\begin{tabular}{lcrrrr}
\hline \hline & $a x+b$ & $z=0$ & $z=0.5$ & $z=1$ & \multicolumn{1}{c}{$z=2$} \\
\hline \multirow{2}{*}{ Sc } & $a$ & $0.66 \pm 0.17$ & $0.76 \pm 0.09$ & $0.89 \pm 0.05$ & $0.91 \pm 0.04$ \\
& $b$ & $-6.55 \pm 0.09$ & $-7.25 \pm 0.05$ & $-8.27 \pm 0.04$ & $-8.20 \pm 0.03$ \\
\hline \multirow{4}{*}{ All galaxies } & $a$ & $0.21 \pm 0.05$ & $0.70 \pm 0.03$ & $0.82 \pm 0.02$ & $0.90 \pm 0.01$ \\
& $b$ & $-2.44 \pm 0.03$ & $-6.76 \pm 0.03$ & $-7.56 \pm 0.02$ & $-8.09 \pm 0.02$ \\
\hline
\end{tabular}

(e.g., Wuyts et al. 2011). However, Fig. 11 (upper panels) shows that current ETGs are not out of the MSSF until relatively recent, $z \leq 0.5$. To determine how this result depends on the spatial sampling, we obtained the $\operatorname{SFR}^{i n}(\mathrm{t})$ and $M_{\star}^{\text {in }}(\mathrm{t})$ for the galaxy regions that today are located in the central 0.5 HLR (lower panels). The lines are the same as in the upper panels. We found that at $z \geq 1$, the inner regions were still on the MSSF and migrate off it at lower redshift. Thus, our results suggest that the massive passive and dead galaxies detected at $z \geq 2$ by high-redshift galaxy surveys cannot be the progenitors of the current ETGs in the CALIFA survey. Spectroscopically confirmed quiescent galaxies at $z>2$ have masses already in excess of $\log M_{\star} \sim 10.5-11$ (e.g., Toft et al. 2012; Belli et al. 2017); thus, if they grow further through mergers, their descendants may be more massive galaxies that are not sampled in the CALIFA volume.

Overall, our results indicate that the MSSF might be a natural consequence of the cold-mode accretion of galaxies
(Birnboim \& Dekel 2003), where the supply of gas feeding the star formation in galaxies follows the dark matter halo accretion rate, with coupled baryons and dark matter halo. However, because the slope is $<1$ even at very high redshift, feedback may also play a role in making the relation sub-linear.

\section{Summary and conclusions}

Using the stellar populations fossil record method for a sample of 366 CALIFA galaxies with GALEX images, we obtained the cosmic evolution of the absolute and specific star formation rate in galaxies, and the galaxy mass. These properties were estimated for galaxies with stellar mass in the range $\sim 10^{9}-10^{12}$ (for a Chabrier IMF) by stacking the results as a function of Hubble type (E, S0, Sa, Sb, Sbc, Sc, and Sd). A Bayesian method based on parametric SFHs that simultaneously fits the FUV, NUV, and $u, g, r, i, z$ bands, and the $\mathrm{D}_{n} 4000, \mathrm{H} \beta$, and [MgFe]' indices from the data cubes are also presented. In the main body of the paper, we discuss the results obtained using a parametric delayed- $\tau$ SFH. In Appendix, the results obtained with other parameterizations (formed by a single or by a combination of two laws) are compared with the delayed- $\tau$ model. Furthermore, we compare these results with those obtained by fitting the $U V$ band and the full CALIFA spectra using a recent version of the nonparametric code STARLIGHT (López Fernández et al. 2016). The fits are processed to derive the time evolution of $\operatorname{SFR}(t)$ and $\operatorname{sSFR}(t)$ for three different regions in each galaxy at the present epoch: a) 0-2 HLR, b) 0-0.5 HLR, and c) 1-2 HLR, 
as representative of the whole (integrated) galaxy, the innermost regions (dominated in most of the galaxies by the spheroidal or bulge component), and outer regions (dominated by the disk in spirals).

Our main results are listed below.

- At any epoch, the SFR scales with the current Hubble type, as expected from the dependence of SFR on $M_{\star}$, and of galaxy mass with morphology. The highest SFR $\left(\sim 40 M_{\odot} \mathrm{yr}^{-1}\right)$ occurred in E galaxies at $z \sim 1-2$. The lowest SFR at similar epochs occurs in late spirals $\mathrm{Sd}\left(\sim 3 M_{\odot} \mathrm{yr}^{-1}\right)$. The SFR in the inner regions of E peaks at $z \sim 2$, while it peaks at $z<1$ in the outer regions. The SFR peak in early spirals ( $\mathrm{Sa}, \mathrm{Sb}$, and $\mathrm{Sbc}$ ) occurred earlier than in $\mathrm{E}$ and $\mathrm{S} 0$. The SFR peak in the inner regions occurred at a similar epoch in E, S0, and early spirals; earlier than for late-type spirals.

- These results are a consequence of the values obtained for the parameters $t_{0}$ and $\tau$. For Sa-Sbc $t_{0} \sim 12 \mathrm{Gyr}, t_{0} \sim 10 \mathrm{Gyr}$ for $\mathrm{E}$ and S0, and $t_{0} \sim 10-9 \mathrm{Gyr}$ in Sc and Sd galaxies. For the present-day inner regions, $t_{0}-\tau$ is longer than that of the whole galaxy and of the present-day outer regions, indicating that the inner regions formed earlier than outer regions, and that galaxies formed inside-out. The e-folding time $\tau$ increases with morphological type and is higher in the outer than in the inner regions of spirals, indicating that star formation is more extended in time in late-type than in early-type spirals.

- The CALIFA sample is well suited to compute the evolution of $\rho_{\text {SFR }}$ and $\rho_{\star}$, which is in agreement with results obtained from galaxy surveys, in particular with the recent estimates obtained from the GAMA, G10-COSMOS, and 3D-HST survey by Driver et al. (2017). At $z \leq 0.5$, the majority of $\rho_{\mathrm{SFR}}$ takes place in the outer regions of galaxies, but at higher redshifts, the present-day inner regions $(<0.5 \mathrm{HLR})$ play a major role in building $\rho_{\mathrm{SFR}}$ and $\rho_{\star}$ dominating at $z>2$. In terms of morphology, while at $z=0$ late spirals dominate the $\rho_{\mathrm{SFR}}$ budget, at $z>2$, the progenitors of the present-day $\mathrm{E}$ and S0 are the main contributors to the SFR density and $\rho_{\star}$. When we take the $50 \%$ point of $\rho_{\star}$ as a reference, the inner regions reached this value at $9 \mathrm{Gyr}$ and the outer regions at $6 \mathrm{Gyr}$ lookback time. This delay between the inner and outer regions confirms that galaxies grow inside-out.

- The sSFR declines rapidly as the Universe evolves, although the slope depends on the morphology; it is steeper for early-type than for late-type galaxies. In the inner regions, the SSFR declines with time more rapidly than in the outer regions in early-type spirals (Sa-Sbc) and E and S0, suggesting an earlier epoch for the shut-down of the star formation.

- The MSSF is traced up to $z>2$. We find that the slope evolves with time, in agreement with cosmological galaxy surveys. The slope flattens from $0.9 \pm 0.01$ at $z=2$ to $0.66 \pm 0.17$ at the current epoch (when only Sc galaxies are considered). Our estimates of the evolution of the MSSF are in good agreement with the predictions by the Illustris simulation. They suggest that the MSSF is a natural consequence of a cold-mode accretion, although feedback may also play some role to set the slope of the correlation below to 1 .

- The comparison of nine different parametric SFHs (described in appendix) indicates that a delayed- $\tau$ SFR model provides a better match between our results and those from the snapshots of galaxy evolution obtained by studies at different redshifts for $\rho_{\mathrm{SFR}}, \operatorname{sSFR}(t)$, and $\rho_{\star}$. The average SFH of galaxies, as represented by $m(t)$ versus lookback time of CALIFA galaxies, confirms that galaxies globally grow their mass mainly in a mode that is well represented by a delayed- $\tau$, where the maximum peaks at high redshift $(z \sim$ 2 ), and then declines exponentially with an e-folding time of $\sim 3.9$ Gyr. This result is in agreement with the model proposed by Madau \& Dickinson (2014) and our previous results using non-parametric SFH (González Delgado et al. 2017).

These results again show the uniqueness of the CALIFA survey in characterizing the the cosmic evolution of the spatially resolved SFR and stellar mass of galaxies. The fossil record of the stellar populations of this sample of nearby galaxies has been very successful in deriving $\rho_{\mathrm{SFR}}, \mathrm{sSFR}$, and $\rho_{\star}$ that agree well with the results from the snapshot galaxy surveys in a wide range of redshifts. The spatially resolved data allowed us to retrieve the contributions of different regions of early-type and spiral galaxies to $\rho_{\mathrm{SFR}}$ and $\rho_{\star}$.

Acknowledgements. CALIFA is the first legacy survey carried out at Calar Alto. The CALIFA collaboration would like to thank the IAA-CSIC and MPIA-MPG as the main partners of the observatory, and CAHA itself, for the unique access to telescope time and support in manpower and infrastructures. We also thank the CAHA staff for the dedication to this project. Support from the Spanish Ministerio de Economía y Competitividad, through projects AYA2016-77846P, AYA2014-57490-P, AYA2010-15081, and the Junta de Andalucía FQ1580 is gratefully acknowledged. We also thank the Viabilidad, Diseño, Acceso y Mejora funding program, ICTS-2009-10, for funding the data acquisition of this project. RCF acknowledges the hospitality of the IAA and the support of CNPq. RGD acknowledges the support of CNPq (Brazil) through Programa Ciência sem Fronteiras (401452/2012-3). RCF and NVA acknowledge the support from the CAPES CsF-PVE project 88881.068116/2014-01. SFS thanks the ConaCyt programs IA180125 and DGAPA IA101217 for their support to this project. We acknowledge the support of the IAA Computing group, and the referee for useful comments.

\section{References}

Ahn, C. P., Alexandroff, R., Allende Prieto, C., et al. 2014, ApJS, 211, 17 Alongi, M., Bertelli, G., Bressan, A., et al. 1993, A\&AS, 97, 851

Arnouts, S., Walcher, C. J., Le Fèvre, O., et al. 2007, A\&A, 476, 137

Asari, N. V., Cid Fernandes, R., Stasińska, G., et al. 2007, MNRAS, 381, 263

Baldry, I. K., Glazebrook, K., \& Driver, S. P. 2008, MNRAS, 388, 945

Baldry, I. K., Robotham, A. S. G., Hill, D. T., et al. 2010, MNRAS, 404, 86

Baldry, I. K., Driver, S. P., Loveday, J., et al. 2012, MNRAS, 421, 621

Balogh, M. L., Morris, S. L., Yee, H. K. C., Carlberg, R. G., \& Ellingson, E. 1999, ApJ, 527, 54

Behroozi, P. S., Wechsler, R. H., \& Conroy, C. 2013, ApJ, 770, 57

Belli, S., Genzel, R., Förster Schreiber, N. M., et al. 2017, ApJ, 841, L6

Birnboim, Y., \& Dekel, A. 2003, MNRAS, 345, 349

Birnboim, Y., Dekel, A., \& Neistein, E. 2007, MNRAS, 380, 339

Blanton, M. R., \& Moustakas, J. 2009, ARA\&A, 47, 159

Bluck, A. F. L., Conselice, C. J., Bouwens, R. J., et al. 2009, MNRAS, 394, L51

Bluck, A. F. L., Conselice, C. J., Buitrago, F., et al. 2012, ApJ, 747, 34

Bourne, N., Dunlop, J. S., Merlin, E., et al. 2017, MNRAS, 467, 1360

Bressan, A., Fagotto, F., Bertelli, G., \& Chiosi, C. 1993, A\&AS, 100, 647

Brinchmann, J., Charlot, S., White, S. D. M., et al. 2004, MNRAS, 351, 1151

Bruzual, A. G., \& Kron, R. G. 1980, ApJ, 241, 25

Bruzual, G., \& Charlot, S. 2003, MNRAS, 344, 1000

Bryant, J. J., Owers, M. S., Robotham, A. S. G., et al. 2015, MNRAS, 447, 2857

Bundy, K., Fukugita, M., Ellis, R. S., et al. 2009, ApJ, 697, 1369

Bundy, K., Bershady, M. A., Law, D. R., et al. 2015, ApJ, 798, 7

Calzetti, D., Armus, L., Bohlin, R. C., et al. 2000, ApJ, 533, 682

Cano-Díaz, M., Sánchez, S. F., Zibetti, S., et al. 2016, ApJ, 821, L26

Cappellari, M. 2016, ARA\&A, 54, 597

Cappellari, M., Emsellem, E., Krajnović, D., et al. 2011, MNRAS, 413, 813

Caputi, K. I., Cirasuolo, M., Dunlop, J. S., et al. 2011, MNRAS, 413, 162

Casteels, K. R. V., Conselice, C. J., Bamford, S. P., et al. 2014, MNRAS, 445, 1157

Catalán-Torrecilla, C., Gil de Paz, A., Castillo-Morales, A., et al. 2015, A\&A, 584, A87

Catalán-Torrecilla, C., Gil de Paz, A., Castillo-Morales, A., et al. 2017, ApJ, 848, 87

Chabrier, G. 2003, PASP, 115, 763

Chiosi, C. 1980, A\&A, 83, 206

Chiosi, C., Sciarratta, M., D’Onofrio, M., et al. 2017, ApJ, 851, 44

Cid Fernandes, R., Mateus, A., Sodré, L., Stasińska, G., \& Gomes, J. M. 2005, MNRAS, 358, 363 
Cid Fernandes, R., Pérez, E., García Benito, R., et al. 2013, A\&A, 557, A86

Cid Fernandes, R., González Delgado, R. M., García Benito, R., et al. 2014, A\&A, 561, A130

Cimatti, A., Daddi, E., Renzini, A., et al. 2004, Nature, 430, 184

Citro, A., Pozzetti, L., Moresco, M., \& Cimatti, A. 2016, A\&A, 592, A19

Cortijo-Ferrero, C., González Delgado, R. M., Pérez, E., et al. 2017, A\&A, 607, A70

Daddi, E., Dickinson, M., Morrison, G., et al. 2007, ApJ, 670, 156

de Amorim, A. L., García-Benito, R., Cid Fernandes, R., et al. 2017, MNRAS, 471,3727

Dekel, A., Birnboim, Y., Engel, G., et al. 2009a, Nature, 457, 451

Dekel, A., Sari, R., \& Ceverino, D. 2009b, ApJ, 703, 785

Delgado-Serrano, R., Hammer, F., Yang, Y. B., et al. 2010, A\&A, 509, A78

Dickinson, M., Papovich, C., Ferguson, H. C., \& Budavári, T. 2003, ApJ, 587, 25

Driver, S. P., Andrews, S. K., da Cunha, E., et al. 2017, MNRAS, 475, 2891

Duncan, K., Conselice, C. J., Mortlock, A., et al. 2014, MNRAS, 444, 2960

Dunne, L., Ivison, R. J., Maddox, S., et al. 2009, MNRAS, 394, 3

Dutton, A. A., van den Bosch, F. C., \& Dekel, A. 2010, MNRAS, 405, 1690

Elbaz, D., Daddi, E., Le Borgne, D., et al. 2007, A\&A, 468, 33

Elbaz, D., Dickinson, M., Hwang, H. S., et al. 2011, A\&A, 533, A119

Fagotto, F., Bressan, A., Bertelli, G., \& Chiosi, C. 1994a, A\&AS, 104, 365

Fagotto, F., Bressan, A., Bertelli, G., \& Chiosi, C. 1994b, A\&AS, 105, 29

Falcón-Barroso, J., Sánchez-Blázquez, P., Vazdekis, A., et al. 2011, A\&A, 532, A95

Fardal, M. A., Katz, N., Weinberg, D. H., \& Davé, R. 2007, MNRAS, 379, 985

Feulner, G., Gabasch, A., Salvato, M., et al. 2005, ApJ, 633, L9

Fontana, A., Poli, F., Menci, N., et al. 2003, ApJ, 587, 544

Gallagher, III, J. S., Hunter, D. A., \& Tutukov, A. V. 1984, ApJ, 284, 544

Gallazzi, A., Charlot, S., Brinchmann, J., \& White, S. D. M. 2006, MNRAS, 370 1106

Gallazzi, A., Brinchmann, J., Charlot, S., \& White, S. D. M. 2008, MNRAS, 383, 1439

Gallego, J., Zamorano, J., Aragon-Salamanca, A., \& Rego, M. 1995, ApJ, 455,

García-Benito, R., Zibetti, S., Sánchez, S. F., et al. 2015, A\&A, 576, A135

García-Benito, R., González Delgado, R. M., Pérez, E., et al. 2017, A\&A, 608 A27

Gavazzi, G., Bonfanti, C., Sanvito, G., Boselli, A., \& Scodeggio, M. 2002, ApJ, 576,135

Girardi, L., Bressan, A., Chiosi, C., Bertelli, G., \& Nasi, E. 1996, A\&AS, 117, 113

Glazebrook, K., Abraham, R. G., McCarthy, P. J., et al. 2004, Nature, 430, 181

Goddard, D., Thomas, D., Maraston, C., et al. 2016, AJ, 152, 197

González, V., Labbé, I., Bouwens, R. J., et al. 2011, ApJ, 735, L34

González Delgado, R. M., Cerviño, M., Martins, L. P., Leitherer, C., \& Hauschildt, P. H. 2005, MNRAS, 357, 945

González Delgado, R. M., Pérez, E., Cid Fernandes, R., et al. 2014, A\&A, 562, A47

González Delgado, R. M., García-Benito, R., Pérez, E., et al. 2015, A\&A, 581, A103

González Delgado, R. M., Cid Fernandes, R., Pérez, E., et al. 2016, A\&A, 590, A44

González Delgado, R. M., Pérez, E., Cid Fernandes, R., et al. 2017, A\&A, 607, A128

Grazian, A., Fontana, A., Santini, P., et al. 2015, A\&A, 575, A96

Gunawardhana, M. L. P., Hopkins, A. M., Bland-Hawthorn, J., et al. 2013 MNRAS, 433, 2764

Gunawardhana, M. L. P., Hopkins, A. M., Taylor, E. N., et al. 2015, MNRAS, 447,875

Heavens, A., Panter, B., Jimenez, R., \& Dunlop, J. 2004, Nature, 428, 625

Hopkins, A. M. \& Beacom, J. F. 2006, ApJ, 651, 142

Husemann, B., Jahnke, K., Sánchez, S. F., et al. 2013, A\&A, 549, A87

Ibarra-Medel, H. J., Sánchez, S. F., Avila-Reese, V., et al. 2016, MNRAS, 463, 2799

Ilbert, O., McCracken, H. J., Le Fèvre, O., et al. 2013, A\&A, 556, A55

Kajisawa, M., Ichikawa, T., Tanaka, I., et al. 2009, ApJ, 702, 1393

Karim, A., Schinnerer, E., Martínez-Sansigre, A., et al. 2011, ApJ, 730, 61

Kauffmann, G., Heckman, T. M., White, S. D. M., et al. 2003, MNRAS, 341, 54

Kaviraj, S., Schawinski, K., Devriendt, J. E. G., et al. 2007, ApJS, 173, 619

Kaviraj, S., Devriendt, J., Dubois, Y., et al. 2015, MNRAS, 452, 2845

Kelz, A., Verheijen, M. A. W., Roth, M. M., et al. 2006, PASP, 118, 129

Kereš, D., Katz, N., Weinberg, D. H., \& Davé, R. 2005, MNRAS, 363, 2

Koleva, M., Prugniel, P., de Rijcke, S., \& Zeilinger, W. W. 2011, MNRAS, 417, 1643

Labbé, I., Oesch, P. A., Bouwens, R. J., et al. 2013, ApJ, 777, L19

Law, D. R., Yan, R., Bershady, M. A., et al. 2015, AJ, 150, 19

Le Borgne, J.-F., Bruzual, G., Pelló, R., et al. 2003, A\&A, 402, 433
Lee, K.-S., Ferguson, H. C., Wiklind, T., et al. 2012, ApJ, 752, 66

Lee, N., Sanders, D. B., Casey, C. M., et al. 2015, ApJ, 801, 80

Lee, S.-K., Ferguson, H. C., Somerville, R. S., Wiklind, T., \& Giavalisco, M. 2010, ApJ, 725, 1644

Lehnert, M. D., Di Matteo, P., Haywood, M., \& Snaith, O. N. 2014, ApJ, 789, L30

Lehnert, M. D., van Driel, W., Le Tiran, L., Di Matteo, P., \& Haywood, M. 2015, A\&A, 577, A112

Li, C. \& White, S. D. M. 2009, MNRAS, 398, 2177

Lilly, S. J., Le Fevre, O., Hammer, F., \& Crampton, D. 1996, ApJ, 460, L1

Lilly, S. J., Carollo, C. M., Pipino, A., Renzini, A., \& Peng, Y. 2013, ApJ, 772 119

Lofthouse, E. K., Kaviraj, S., Conselice, C. J., Mortlock, A., \& Hartley, W. 2017, MNRAS, 465, 2895

López Fernández, R., Cid Fernandes, R., González Delgado, R. M., et al. 2016, MNRAS, 458, 184

López-Sanjuan, C., Le Fèvre, O., Ilbert, O., et al. 2012, A\&A, 548, A7

López-Sanjuan, C., Cenarro, A. J., Varela, J., et al. 2015, A\&A, 576, A53

Lotz, J. M., Jonsson, P., Cox, T. J., et al. 2011, ApJ, 742, 103

Madau, P., \& Dickinson, M. 2014, ARA\&A, 52, 415

Madau, P., Pozzetti, L., \& Dickinson, M. 1998, ApJ, 498, 106

Magdis, G. E., Elbaz, D., Daddi, E., et al. 2010, ApJ, 714, 1740

Man, A. W. S., Toft, S., Zirm, A. W., Wuyts, S., \& van der Wel, A. 2012, ApJ, 744,85

Marchesini, D., van Dokkum, P. G., Förster Schreiber, N. M., et al. 2009, ApJ, 701,1765

Martin, D. C., Fanson, J., Schiminovich, D., et al. 2005, ApJ, 619, L1

Martin, G., Kaviraj, S., Devriendt, J. E. G., et al. 2017, MNRAS, 472, L50

Martins, L. P., González Delgado, R. M., Leitherer, C., Cerviño, M., \& Hauschildt, P. 2005, MNRAS, 358, 49

McDermid, R. M., Alatalo, K., Blitz, L., et al. 2015, MNRAS, 448, 3484

Minchev, I., Chiappini, C., Martig, M., et al. 2014, ApJ, 781, L20

Morrissey, P., Conrow, T., Barlow, T. A., et al. 2007, ApJS, 173, 682

Moustakas, J., Coil, A. L., Aird, J., et al. 2013, ApJ, 767, 50

Moustakas, L. A. \& Metcalf, R. B. 2003, MNRAS, 339, 607

Muzzin, A., Marchesini, D., Stefanon, M., et al. 2013, ApJ, 777, 18

Naab, T. \& Ostriker, J. P. 2016, ARA\&A, 55, 59

Neistein, E., \& Dekel, A. 2008, MNRAS, 388, 1792

Neistein, E., van den Bosch, F. C., \& Dekel, A. 2006, MNRAS, 372, 933

Nelson, D., Pillepich, A., Genel, S., et al. 2015, Astron. Comput., 13, 12

Noeske, K. G., Faber, S. M., Weiner, B. J., et al. 2007, ApJ, 660, L47

Ocvirk, P., Pichon, C., Lançon, A., \& Thiébaut, E. 2006, MNRAS, 365, 74

Oliver, S., Frost, M., Farrah, D., et al. 2010, MNRAS, 405, 2279

Pannella, M., Carilli, C. L., Daddi, E., et al. 2009, ApJ, 698, L116

Panter, B., Heavens, A. F., \& Jimenez, R. 2003, MNRAS, 343, 1145

Panter, B., Jimenez, R., Heavens, A. F., \& Charlot, S. 2007, MNRAS, 378 1550

Panter, B., Jimenez, R., Heavens, A. F., \& Charlot, S. 2008, MNRAS, 391, 1117

Peng, Y.-j., Lilly, S. J., Kovač, K., et al. 2010, ApJ, 721, 193

Pérez, E., Cid Fernandes, R., González Delgado, R. M., et al. 2013, ApJ, 764, L1

Pérez-González, P. G., Rieke, G. H., Villar, V., et al. 2008, ApJ, 675, 234

Pozzetti, L., Bolzonella, M., Zucca, E., et al. 2010, A\&A, 523, A13

Reddy, N. A. \& Steidel, C. C. 2009, ApJ, 692, 778

Renzini, A. \& Peng, Y.-j. 2015, ApJ, 801, L29

Rodighiero, G., Vaccari, M., Franceschini, A., et al. 2010, A\&A, 515, A8

Rodriguez-Gomez, V., Genel, S., Vogelsberger, M., et al. 2015, MNRAS, 449, 49

Roškar, R., Debattista, V. P., Quinn, T. R., Stinson, G. S., \& Wadsley, J. 2008, ApJ, 684, L79

Roth, M. M., Kelz, A., Fechner, T., et al. 2005, PASP, 117, 620

Rudnick, G., Rix, H.-W., Franx, M., et al. 2003, ApJ, 599, 847

Salim, S., Charlot, S., Rich, R. M., et al. 2004, in American Astronomical Society Meeting Abstracts, Bull. Am. Astron. Soc., 36, 1411

Salim, S., Rich, R. M., Charlot, S., et al. 2007, ApJS, 173, 267

Sánchez, S. F., Kennicutt, R. C., Gil de Paz, A., et al. 2012, A\&A, 538, A8

Sánchez, S. F., García-Benito, R., Zibetti, S., et al. 2016a, A\&A, 594, A36

Sánchez, S. F., Pérez, E. Sánchez-Blázquez, P., et al. 2016b, Rev. Mex. Astron. Astrofís., 52, 171

Sánchez-Blázquez, P., Peletier, R. F., Jiménez-Vicente, J., et al. 2006, MNRAS, 371, 703

Sánchez-Blázquez, P., Rosales-Ortega, F., Diaz, A., \& Sánchez, S. F. 2014a, MNRAS, 437, 1534

Sánchez-Blázquez, P., Rosales-Ortega, F. F., Méndez-Abreu, J., et al. 2014b, A\&A, 570, A6

Sandage, A. 1986, A\&A, 161, 89

Schiminovich, D., Wyder, T. K., Martin, D. C., et al. 2007, ApJS, 173, 315

Schmidt, M. 1968, ApJ, 151, 393

Searle, L., Sargent, W. L. W., \& Bagnuolo, W. G. 1973, ApJ, 179, 427 
Simha, V., Weinberg, D. H., Conroy, C., et al. 2014, ArXiv e-prints [arXiv: 1404.0402]

Song, M., Finkelstein, S. L., Ashby, M. L. N., et al. 2016, ApJ, 825, 5

Sparre, M., Hayward, C. C., Springel, V., et al. 2015, MNRAS, 447, 3548

Speagle, J. S., Steinhardt, C. L., Capak, P. L., \& Silverman, J. D. 2014, ApJS, 214,15

Stark, D. P., Ellis, R. S., Bunker, A., et al. 2009, ApJ, 697, 1493

Stark, D. P., Schenker, M. A., Ellis, R., et al. 2013, ApJ, 763, 129

Stoughton, C., Lupton, R. H., Bernardi, M., et al. 2002, AJ, 123, 485

Tasca, L. A. M., Le Fèvre, O., Hathi, N. P., et al. 2015, A\&A, 581, A54

Thomas, D., Maraston, C., \& Bender, R. 2003, MNRAS, 339, 897

Tinsley, B. M. 1968, ApJ, 151, 547

Tinsley, B. M. 1972, A\&A, 20, 383

Toft, S., Gallazzi, A., Zirm, A., et al. 2012, ApJ, 754, 3

Tojeiro, R., Percival, W. J., Heavens, A. F., \& Jimenez, R. 2011, MNRAS, 413 , 434

Tomczak, A. R., Quadri, R. F., Tran, K.-V. H., et al. 2016, ApJ, 817, 118
Trager, S. C., Faber, S. M., Worthey, G., \& González, J. J. 2000, AJ, 119, 1645

Vazdekis, A., Sánchez-Blázquez, P., Falcón-Barroso, J., et al. 2010, MNRAS, 404, 1639

Verheijen, M. A. W., Bershady, M. A., Andersen, D. R., et al. 2004, Astron. Nachr., 325, 151

Walcher, C. J., Wisotzki, L., Bekeraité, S., et al. 2014, A\&A, 569, A1

Walcher, J., Groves, B., Budavári, T., \& Dale, D. 2011, Ap\&SS, 331, 1

Weinmann, S. M., Neistein, E., \& Dekel, A. 2011, MNRAS, 417, 2737

Whitaker, K. E., van Dokkum, P. G., Brammer, G., \& Franx, M. 2012, ApJ, 754, L29

Williams, R. J., Quadri, R. F., \& Franx, M. 2011, ApJ, 738, L25

Worthey, G. 1994, ApJS, 95, 107

Wuyts, S., Förster Schreiber, N. M., van der Wel, A., et al. 2011, ApJ, 742, 96

Wyder, T. K., Martin, D. C., Schiminovich, D., et al. 2007, ApJS, 173, 293

Yabe, K., Ohta, K., Iwata, I., et al. 2009, ApJ, 693, 507

Zibetti, S., Gallazzi, A. R., Ascasibar, Y., et al. 2017, MNRAS, 468, 1902 


\section{Appendix A: Comparing results from parametric and non-parametric SFHs}

In this appendix we compare the SFHs obtained with the delayed- $\tau$ model with those obtained with other models, parametric and non-parametric (the STARLIGHT code). We express the SFH by the mass fraction, $m(t)$, which is defined for each galaxy as the ratio of the mass formed in each epoch to the total mass of the galaxy today. We also derive the mass growth curve (see, e.g., Pérez et al. 2013; García-Benito et al. 2017), which provides useful information about how the mass is assembled in a galaxy as a whole and in different regions. From these curves, we compare the epoch at which galaxies assembled $80 \%$ of their current mass, $t_{80}$. Finally, we estimate $\rho_{\mathrm{SFR}}, \rho_{\star}$, and SSFR at different redshifts, and we compare the results from the different models with those from surveys at different redshifts. The goal of this appendix is to show that the delayed- $\tau$ model is the most simple, meaningful, and representative parameterization of the SFHs.

\section{A.1. delayed- $\tau$ versus non-parametric SFH with STARLIGHT}

Our previous analysis obtained with STARLIGHT for the CALIFA sample presented in González Delgado et al. (2017) was made by fitting only the CALIFA spectra, using composite stellar population models built with SSPs by Vazdekis et al. (2010) and González Delgado et al. (2005), assuming a Salpeter IMF. In terms of $m(t)$, we obtain that the highest mass fractions invariably occur at the earliest times, and subsequent star formation varies systematically with $M_{\star}$ and morphology, with the low $M_{\star}$ and also the later spiral galaxies forming stars over more extended periods of time, while high $M_{\star}$ and early-type galaxies exhibit the fastest decline in $m(t)$.

In order to be consistent with the data, here we use STARLIGHT, but now fitting the GALEX (FUV, NUV) photometry and the full CALIFA spectra ${ }^{7}$. The fits were made with the new version of STARLIGHT by López Fernández et al. (2016) in combination with the same collection of SSPs by Charlot \& Bruzual (2007, priv. comm.) used with M1. We also assumed a Chabrier (2003) IMF.

To quantify the differences between the STARLIGHT and the delayed- $\tau$ model, we have discretized $m(t)$ in a few relevant age ranges. Fig. A.1 presents the results for four age ranges: $t \leq 1 \mathrm{Gyr}$ (blue), $1<t \leq 4$ Gyr (green), $4<t \leq 9$ Gyr (yellow), and $t \geq 9$ Gyr (red). Each panel shows three bar charts corresponding to the spatial regions $R<0.5$ HLR (left), the whole galaxy (middle), and the outer regions $1<R<2$ HLR. The results from the delayed- $\tau$ model (M1) and STARLIGHT (ST) are presented in the middle and left columns.

As with ST, most of the stellar mass in M1 is formed very early on and with very little mass in stars younger than 1 Gyr. We note that the main differences occur in a similar way for all the morphological types. Thus, we can conclude that with ST non-parametric SFH, we derive a larger fraction of old stellar populations $(t>9 \mathrm{Gyr}$ and/or $4<t<9 \mathrm{Gyr})$ and fewer intermediate and young components $(1<t<4 \mathrm{Gyr}$ and/or $t<1 \mathrm{Gyr})$. To comment on these differences in more detail, we discuss the results for the inner regions.

$-m(t \leq 1)$. This mass fraction increases from $\mathrm{E}$ to $\mathrm{Sd}$, although the fraction is lower in the inner than in the outer regions. With $\mathrm{ST}, m(t \leq 1) \leq 1 \%$ in Sbc and earlier types,

\footnotetext{
7 Instead of GALEX and SDSS photometry and the spectroscopic indexes, $\mathrm{D}_{n} 4000, \mathrm{H} \beta$, and [FeMg]' used in M1.
}
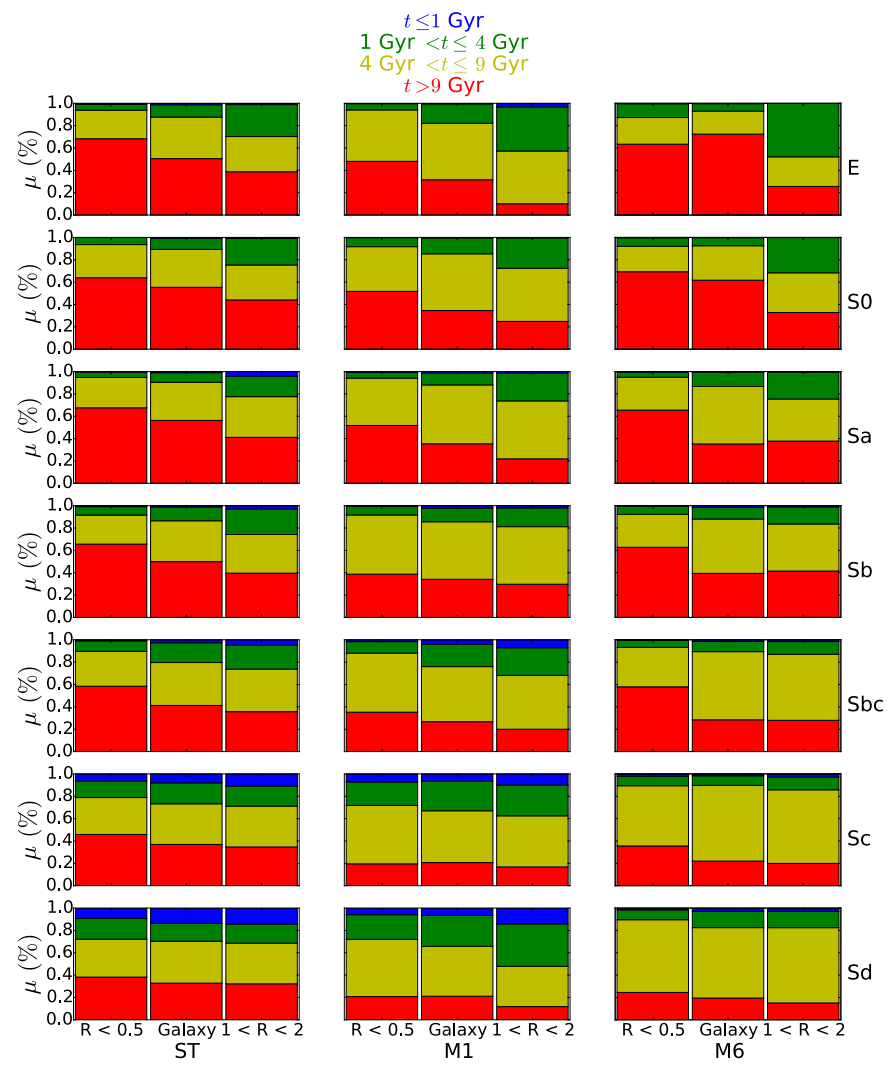

Fig. A.1. Results of mass fraction $m(t)$ grouped in four different age ranges (color-coded as indicated at the top) from STARLIGHT (ST, left), the delayed- $\tau$ model (M1, middle), and a two-exponential model (M6, right).

increasing to $m(t \leq 1) \sim 6 \%$ and $9 \%$ for Sc and Sd, respectively. Using M1, $m(t \leq 1) \leq 2 \%$ in Sbc and earlier types increases to $m(t \leq 1) \sim 7 \%$ and $6 \%$ for Sc and Sd galaxies, respectively.

- $m(1<t \leq 4)$. On average, we obtain lower mass fractions of this component in the inner than in the outer regions for all the Hubble types and all the models. Using ST and M1, the mass fraction of this component increases from early-type to late-type galaxies. For E galaxies, $m(1<t \leq 4) \sim 5 \%$ and $6 \%$, for ST and M1, respectively. On the other hand, for Sd galaxies, $m(1<t \leq 4) \sim 19 \%$ and $22 \%$, respectively.

- $m(4<t \leq 9)$. With ST and M1, we obtain similar mass fractions of this component for all the Hubble types. With ST, $20 \% \leq m(4<t \leq 9) \leq 30 \%$. For M1, we obtain $3 \% \leq$ $m(4<t \leq 9) \leq 46 \%$ for E, S0, and Sa galaxies; $m(4<t \leq 9)$ $\sim 53 \%$ for $\mathrm{Sb}$, Sbc, and Sc; and $m(4<t \leq 9) \sim 52 \%$ for $\mathrm{Sd}$ galaxies.

- $m(t>9)$. For all the models, the old component decreases from $\mathrm{E}$ to $\mathrm{Sd}$ galaxies and is larger in the inner region. For E galaxies, $m(t>9) \sim 74 \%$ and $48 \%$, for ST and M1, respectively. For Sd galaxies, $m(t>9) \sim 42 \%$ and $21 \%$, respectively.

Other interesting results come from the comparison of the epoch at which galaxies assemble $80 \%$ of their mass, $t_{80}$. Based on our previous analysis with STARLIGHT, Pérez et al. (2013) and García-Benito et al. (2017) found that the most massive galaxies assembled their mass earlier than the low-mass galaxies, a signature of downsizing. We have also obtained that $t_{80}$ for the inner regions is higher than for the outer regions, suggesting that galaxies assemble their mass inside-out. 


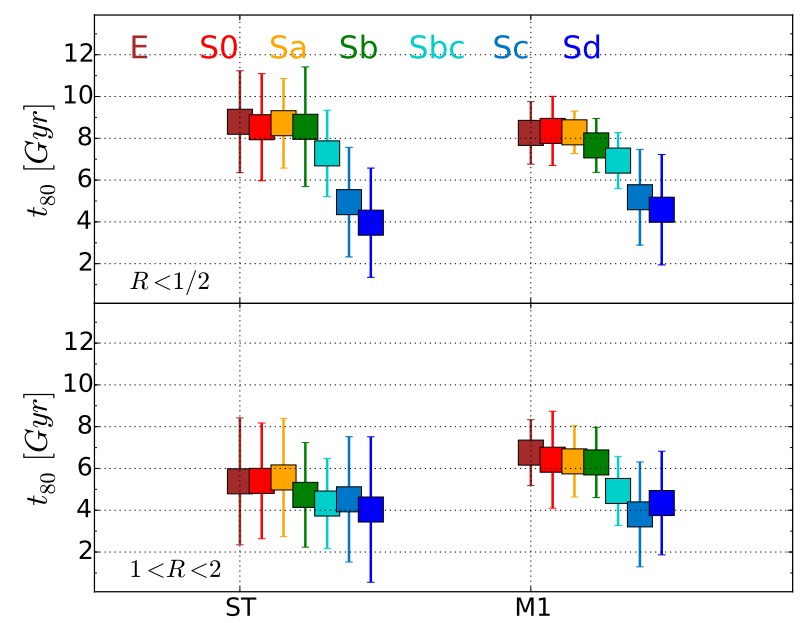

Fig. A.2. Lookback time at which galaxies assemble $80 \%$ of their mass, $t_{80}$, from STARLIGHT and from M1, corresponding to the inner $R<0.5$ HLR and outer regions $1<R<2$ HLR.

Figure A. 2 compares the results from M1 and ST for $t_{80}$ in the inner and outer regions. They are listed below.

- Inner regions. 1) M1, $t_{80}$ decreases with Hubble type, with E, S0, and Sa having similar values, $t_{80} \sim 9-8$ Gyr, while $t_{80} \sim 5$ Gyr for Sc and Sd. 2) The results with ST are very similar, $t_{80}$ decreasing from early-type to late-type galaxies. For Sb and earlier types, $t_{80} \sim 8-9 \mathrm{Gyr}, t_{80} \sim 5 \mathrm{Gyr}$ for Sc, and $t_{80} \sim 4 \mathrm{Gyr}$ for Sd, slightly lower than with M1.

- Outer regions. 1) M1, $t_{80}$ decreases with the morphology, lower for later types. The values for the outer regions are lower than for the inner regions for all types. For $\mathrm{Sb}$ and earlier types, $t_{80} \sim 6-7 \mathrm{Gyr}$, and for Sd galaxies $t_{80} \sim 4$ Gyr. 2) With ST, $t_{80}$ decreases similarly with Hubble type, from $t_{80} \sim 5-6 \mathrm{Gyr}$ for $\mathrm{Sa}$ and later types to $t_{80} \sim 4 \mathrm{Gyr}$ for $\mathrm{Sd}$.

\section{A.2. delayed- $\tau$ versus other parametric SFHs}

Other parametric SFHs have been used to fit the observational constrains. They are of two types:

a) One single function, as in the $\tau$ model:

- A $\tau$ model (M2):

$\psi(t)=\psi_{0} \mathrm{e}^{-\left(t_{0}-t\right) / \tau}$

- Sandage model (Sandage 1986; M3):

$$
\psi(t)=\frac{A}{\tau^{2}}\left(t_{0}-t\right) \mathrm{e}^{-\left(t_{0}-t\right)^{2} / 2 \tau^{2}}
$$

- Linearly rising (M4):

$$
\psi(t)=\psi_{0}-\frac{d \psi}{d t}\left(t_{0}-t\right)
$$

- Power rising (M5):

$$
\psi(t)=\psi_{0}\left(t_{0}-t\right)^{\alpha}
$$

b) A combination of two functions:

- Two exponentials (M6):

$$
\begin{aligned}
& \psi_{\text {old }}(t)=\psi_{0} \mathrm{e}^{-\left(t_{0}^{\text {old }}-t\right) / \tau^{\text {old }}} \\
& \psi_{\text {young }}(t)=\psi_{0} \mathrm{e}^{-\left(t_{0}^{\text {young }}-t\right) / \tau^{\text {young }}}
\end{aligned}
$$

- Two exponentials, with the old component fixed (M7):

$$
\begin{aligned}
& \psi_{\text {old }}(t)=\psi_{0} \mathrm{e}^{-(14 \mathrm{Gyr}-t) / \tau^{\text {old }}} \\
& \psi_{\text {young }}(t)=\psi_{0} \mathrm{e}^{-\left(t_{0}^{\text {young }}-t\right) / \tau^{\text {young }}}
\end{aligned}
$$

- An exponential plus a Sandage component (M8):

$$
\begin{aligned}
& \psi_{\text {old }}(t)=\psi_{0} \mathrm{e}^{-\left(t_{0}^{\text {old }}-t\right) / \tau^{\text {old }}} \\
& \psi_{\text {young }}(t)=\frac{A}{\left[\tau^{\text {young }}\right]^{2}}\left(t_{0}^{\text {young }}-t\right) \mathrm{e}^{-\left(t_{0}^{\text {young }}-t\right)^{2} / 2\left[\tau^{\text {young }}\right]^{2}}
\end{aligned}
$$

- Constant SFR plus an exponential declining (M9):

$$
\begin{aligned}
& \psi_{\text {old }}(t)=\psi_{0} \\
& \psi_{\text {young }}(t)=\psi_{0} \mathrm{e}^{-\left(t_{0}-t\right) / \tau}
\end{aligned}
$$

Figure A.3 shows the quality of the fits; we conclude that a) M2 and M3 provide similar quality in the fits as M1; b) M4 and M5 give very poor fits; c) the quality of the fits with M6, M7, and M8 is somewhat better than with M1; and d) M9 provides fits of similar quality to M1. Although M1 is not the model with the lowest $\chi^{2}$, the results related to the SFH of galaxies and stellar population properties are better than with the other models, as explained below; we therefore use M1 as the reference model.

Figure A.1 shows a detailed comparison between M6, M1, and STARLIGHT. Table A.1 lists the results for all the models.

To show further differences and similarities between the parametric models and STARLIGHT, Fig. A.4 shows $t_{80}$ calculated for the whole galaxy ( $R<2$ HLR). The results are averaged by Hubble type.

- Except for M4, the parametric and non-parametric models show that $t_{80}$ decreases with Hubble type. This is a manifestation of the downsizing scenario since in our sample the galaxy mass scales on average with Hubble type. We note, however, that the range of variation from $\mathrm{E}$ to $\mathrm{Sd}$ is smaller with M5 and M9.

- With STARLIGHT, $t_{80} \sim 7$ Gyr for Sa and earlier types and decreases for later types, down to $t_{80} \sim 3.5 \mathrm{Gyr}$ for $\mathrm{Sd}$. Results with M1 are very similar to STARLIGHT for Sa and earlier types, with $t_{80} \sim 7 \mathrm{Gyr}$; but for later types, Sd, STARLIGHT gives a shorter $t_{80}$ than M1.

- For M2, $t_{80} \sim 8$ Gyr for S0 and Sb, and larger for Sa. For E, $t_{80} \sim 7.5 \mathrm{Gyr}$ and lower values are obtained for later spirals, with $t_{80} \sim 5.5$ Gyr for Sd.

- M3 gives lower values than M1. For Sa and earlier types, $t_{80} \sim 6-7 \mathrm{Gyr}, t_{80} \sim 6 \mathrm{Gyr}$ for Sb galaxies, and $t_{80} \sim 4 \mathrm{Gyr}$ for Sc and Sd.

- Results with M6 and M8 are very similar. In both cases, $t_{80}$ decreases from $10 \mathrm{Gyr}$ in E galaxies to 6-7 Gyr for Sc and $\mathrm{Sd}$.

- With M7, $t_{80}$ is larger for all the Hubble types. For Sd $t_{80} \sim$ $10 \mathrm{Gyr}$, which is the value obtained for E galaxies with M6 and M8. For E and S0 galaxies, $t_{80} \sim 12-13$ Gyr.

- The range of variation of $t_{80}$ with M9 is lower than with the other models. For Sb and earlier types, $t_{80} \sim 8 \mathrm{Gyr}$, while for Sc and $\mathrm{Sd} t_{80} \sim 7 \mathrm{Gyr}$. 


\section{A.3. Fossil cosmology versus galaxy surveys: $\rho_{\mathrm{SFR}}, s S F R$, and $\rho_{\star}$}

Finally we compare the results from the different models with fossil cosmology with respect to galaxy surveys, calculating $\rho_{\mathrm{SFR}}, \mathrm{sSFR}$, and $\rho_{\star}$ as a function of redshift. In Sect. 5 we compared these properties for model M1 with results from galaxy surveys from the literature. Here we extend the comparisons to all the parametric models presented in this appendix. From Fig. A.5, we conclude the following.

- M2. The $\rho_{\mathrm{SFR}}$ increases with redshift, and it does not show a maximum (or plateau) at $z \sim 2$ as the cosmological galaxy surveys do. $\rho_{\star}$ increases with time more rapidly than any other single parametric SFH, and the galaxy surveys. The $\operatorname{sSFR}(t)$ for $z>0.5$ is below the lower envelope of points from the cosmological galaxy surveys.

- M3. The $\rho_{\mathrm{SFR}}$ has the maximum at intermediate redshift $z \sim 1$. The evolution of $\operatorname{sSFR}(t)$ is very similar to model M1 and the individual measurements from galaxy surveys. However, $\rho_{\star}$ increases more slowly than in M1 and the galaxy surveys, although it reaches a similar value as M1 at $z=0$.

- M4 and M5. They give very unrealistic results for the SFH of nearby galaxies, and are not able to fit the observational constraints well (see Fig. A.3). Moreover, $\rho_{\mathrm{SFR}}, \rho_{\star}$, and $\operatorname{sSFR}(t)$ do not match the results from galaxy surveys. At $\mathrm{z}=$ 0 , the $\rho_{\mathrm{SFR}}$ with these models is significantly higher than in cosmological galaxy surveys, and at $z \geq 1$ it is significantly lower. The $\operatorname{sSFR}(t)$ evolves with time more smoothly than galaxy surveys do. M4 gives a cosmic evolution of $\rho_{\star}$ that does not match the points from galaxy surveys at all; and M5 gives values of $\rho_{\star}$ at intermediate redshift that are not in agreement with results from the galaxy surveys.

- M6 and M8. The $\rho_{\mathrm{SFR}}, \operatorname{sSFR}(t)$, and $\rho_{\star}$ are similar to galaxy surveys, except that the $\operatorname{sSFR}(t)$ at $z \geq 2$ is lower than the values of the literature. These models not provide an $m(t)$ that is very different to that obtained by Madau \& Dickinson (2014; see Fig. 9).

- M7. The fraction in stars older than 9 Gyr is significantly higher than for the other models, and thus the galaxies grow their mass very rapidly and very early on. As a consequence, the fraction of stellar mass in components of intermediate ages are low. Thus, although at $z=0$ and $z \sim 4$ the $\rho_{\mathrm{SFR}}$ is similar to cosmological galaxy surveys, its evolution is quite different to the results from these works, since a minimum occurs at intermediate redshifts. Similarly, the $\operatorname{sSFR}(t)$ for $z>0.5$ is quite low in comparison with the galaxy surveys. In contrast, $\rho_{\star}$ does not evolve with time; it is almost constant since $z \leq 5$.

- M9. The evolution of $\rho_{\mathrm{SFR}}$ up to $z=2$ is similar to the galaxy surveys, but it does not show a change in slope at $z>2$ and continues to increase with redshift. In absolute values, $\rho_{\mathrm{SFR}} \sim 0.2-0.3$ dex below the Madau \& Dickinson (2014) curve. The $\operatorname{sSFR}(t)$ is significantly below the curve

$$
\Delta=\frac{\text { Synthetic-Observed }}{\text { Error }}
$$

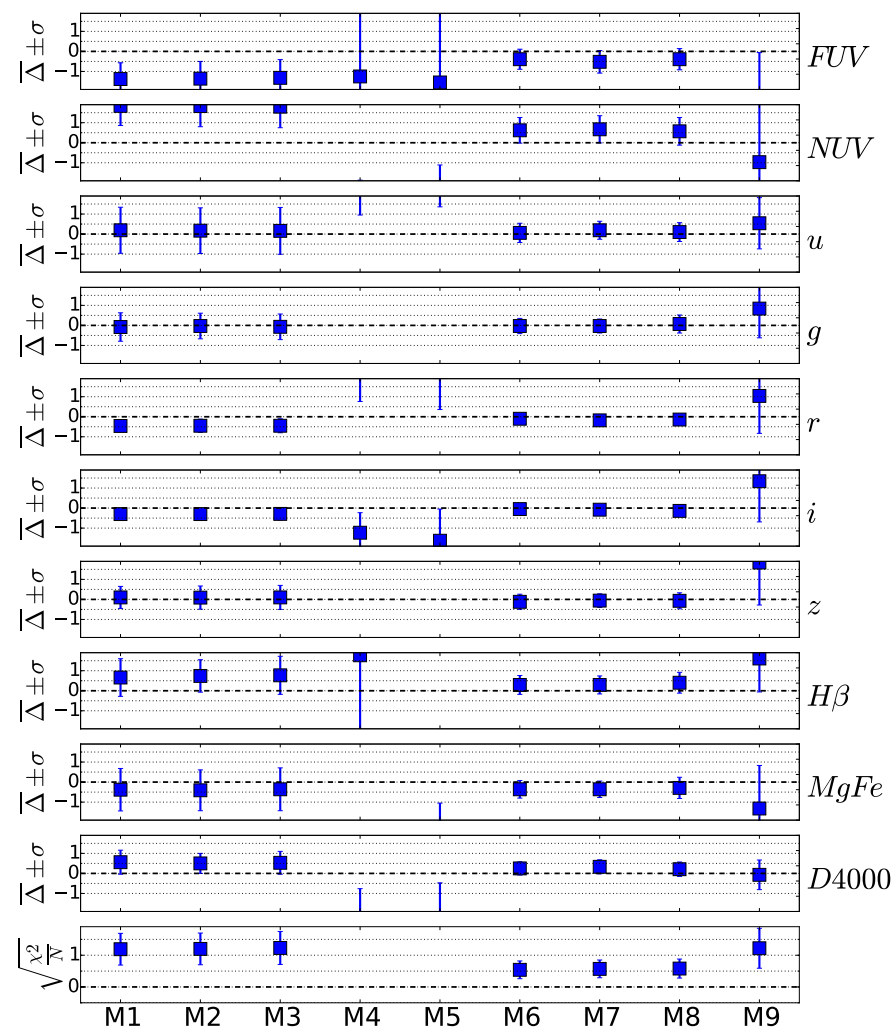

Fig. A.3. Quality of the fits using the different models. Each panel shows $\Delta \pm \sigma$ for each of the observables. $\Delta$ is the average of the difference between the synthetic and the observable quantity divided by the error. The bottom panel, however, shows the reduced $\chi^{2}$.

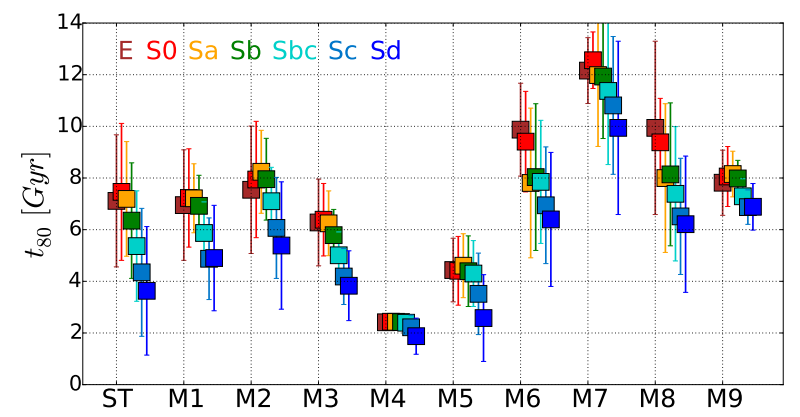

Fig. A.4. Lookback time at which galaxies assemble $80 \%$ of their mass, $t_{80}$, from the different parametric models and STARLIGHT. The average values are color-coded as a function of morphology.

reported by Elbaz et al. (2011); this is also a consequence of the rapid growth of $\rho_{\star}$. 
Table A.1. Average mass fraction, corresponding to the whole galaxy, due to stars in different age ranges as a function of Hubble type, obtained with STARLIGHT and with the parametric models.

\begin{tabular}{|c|c|c|c|c|c|c|c|c|}
\hline & & $\mathrm{E}$ & So & $\mathrm{Sa}$ & $\mathrm{Sb}$ & Sbc & $\mathrm{Sc}$ & $\mathrm{Sd}$ \\
\hline \multirow{4}{*}{ ST } & $m_{t<1 \mathrm{Gyr}}$ & $1 \pm 3$ & $1 \pm 1$ & $1 \pm 1$ & $1 \pm 1$ & $2 \pm 2$ & $7 \pm 14$ & $13 \pm 18$ \\
\hline & $m_{1<t<4 \mathrm{Gyr}}$ & $11 \pm 11$ & $10 \pm 14$ & $9 \pm 8$ & $12 \pm 10$ & $18 \pm 14$ & $19 \pm 16$ & $16 \pm 14$ \\
\hline & $m_{4<t<9 \mathrm{Gyr}}$ & $30 \pm 21$ & $28 \pm 18$ & $29 \pm 16$ & $32 \pm 17$ & $35 \pm 12$ & $33 \pm 15$ & $34 \pm 15$ \\
\hline & $m_{t>9 \mathrm{Gyr}}$ & $58 \pm 25$ & $61 \pm 23$ & $61 \pm 20$ & $54 \pm 22$ & $45 \pm 19$ & $40 \pm 20$ & $36 \pm 19$ \\
\hline \multirow{4}{*}{ M1 } & $m_{t<1 \mathrm{Gyr}}$ & $1 \pm 1$ & $1 \pm 1$ & $1 \pm 1$ & $2 \pm 2$ & $4 \pm 2$ & $6 \pm 3$ & $7 \pm 4$ \\
\hline & $m_{1<t<4 \mathrm{Gyr}}$ & $17 \pm 31$ & $14 \pm 27$ & $10 \pm 13$ & $12 \pm 11$ & $20 \pm 14$ & $26 \pm 17$ & $28 \pm 21$ \\
\hline & $m_{4<t<9 \mathrm{Gyr}}$ & $51 \pm 30$ & $51 \pm 28$ & $53 \pm 20$ & $51 \pm 16$ & $49 \pm 10$ & $46 \pm 10$ & $45 \pm 15$ \\
\hline & $m_{t>9 \mathrm{Gyr}}$ & $32 \pm 28$ & $35 \pm 27$ & $36 \pm 21$ & $34 \pm 17$ & $27 \pm 11$ & $21 \pm 12$ & $21 \pm 17$ \\
\hline \multirow{4}{*}{ M2 } & $m_{t<1 \mathrm{Gyr}}$ & $1 \pm 1$ & $1 \pm 1$ & $1 \pm 1$ & $2 \pm 2$ & $3 \pm 1$ & $8 \pm 18$ & $6 \pm 4$ \\
\hline & $m_{1<t<4 \mathrm{Gyr}}$ & $16 \pm 20$ & $11 \pm 26$ & $9 \pm 14$ & $10 \pm 12$ & $16 \pm 16$ & $20 \pm 19$ & $30 \pm 27$ \\
\hline & $m_{4<t<9 \mathrm{Gyr}}$ & $47 \pm 26$ & $44 \pm 24$ & $39 \pm 23$ & $37 \pm 16$ & $37 \pm 10$ & $37 \pm 13$ & $39 \pm 23$ \\
\hline & $m_{t>9 \mathrm{Gyr}}$ & $37 \pm 27$ & $44 \pm 25$ & $51 \pm 26$ & $51 \pm 20$ & $48 \pm 14$ & $35 \pm 17$ & $25 \pm 24$ \\
\hline \multirow{4}{*}{ M3 } & $m_{t<1 \mathrm{Gyr}}$ & $1 \pm 1$ & $1 \pm 1$ & $1 \pm 1$ & $2 \pm 2$ & $5 \pm 2$ & $7 \pm 3$ & $8 \pm 4$ \\
\hline & $m_{1<t<4 \mathrm{Gyr}}$ & $18 \pm 28$ & $15 \pm 23$ & $14 \pm 11$ & $18 \pm 15$ & $24 \pm 12$ & $29 \pm 11$ & $33 \pm 17$ \\
\hline & $m_{4<t<9 \mathrm{Gyr}}$ & $61 \pm 27$ & $60 \pm 20$ & $62 \pm 15$ & $59 \pm 14$ & $54 \pm 9$ & $50 \pm 8$ & $46 \pm 14$ \\
\hline & $m_{t>9 \mathrm{Gyr}}$ & $21 \pm 20$ & $24 \pm 16$ & $23 \pm 16$ & $21 \pm 9$ & $17 \pm 6$ & $14 \pm 6$ & $12 \pm 7$ \\
\hline \multirow{4}{*}{ M4 } & $m_{t<1 \mathrm{Gyr}}$ & $15 \pm 1$ & $15 \pm 1$ & $15 \pm 1$ & $15 \pm 1$ & $15 \pm 2$ & $18 \pm 12$ & $24 \pm 19$ \\
\hline & $m_{1<t<4 \mathrm{Gyr}}$ & $38 \pm 2$ & $38 \pm 1$ & $38 \pm 1$ & $38 \pm 1$ & $38 \pm 3$ & $40 \pm 8$ & $42 \pm 12$ \\
\hline & $m_{4<t<9 \mathrm{Gyr}}$ & $38 \pm 2$ & $38 \pm 1$ & $38 \pm 1$ & $38 \pm 1$ & $38 \pm 3$ & $35 \pm 9$ & $29 \pm 14$ \\
\hline & $m_{t>9 \mathrm{Gyr}}$ & $9 \pm 1$ & $9 \pm 1$ & $9 \pm 1$ & $9 \pm 1$ & $9 \pm 1$ & $7 \pm 3$ & $5 \pm 4$ \\
\hline \multirow{4}{*}{ M5 } & $m_{t<1 \mathrm{Gyr}}$ & $10 \pm 5$ & $14 \pm 20$ & $13 \pm 18$ & $14 \pm 21$ & $11 \pm 12$ & $16 \pm 18$ & $25 \pm 24$ \\
\hline & $m_{1<t<4 \mathrm{Gyr}}$ & $29 \pm 11$ & $25 \pm 7$ & $25 \pm 8$ & $25 \pm 8$ & $28 \pm 10$ & $32 \pm 12$ & $379 \pm 16$ \\
\hline & $m_{4<t<9 \mathrm{Gyr}}$ & $39 \pm 10$ & $38 \pm 11$ & $38 \pm 10$ & $38 \pm 10$ & $39 \pm 10$ & $36 \pm 11$ & $29 \pm 16$ \\
\hline & $m_{t>9 \mathrm{Gyr}}$ & $22 \pm 11$ & $23 \pm 10$ & $25 \pm 8$ & $23 \pm 10$ & $21 \pm 11$ & $15 \pm 12$ & $9 \pm 11$ \\
\hline \multirow{4}{*}{ M6 } & $m_{t<1 \mathrm{Gyr}}$ & $1 \pm 1$ & $1 \pm 1$ & $1 \pm 4$ & $2 \pm 5$ & $2 \pm 3$ & $2 \pm 3$ & $3 \pm 7$ \\
\hline & $m_{1<t<4 \mathrm{Gyr}}$ & $11 \pm 17$ & $9 \pm 12$ & $14 \pm 22$ & $11 \pm 21$ & $8 \pm 21$ & $8 \pm 20$ & $14 \pm 23$ \\
\hline & $m_{4<t<9 \mathrm{Gyr}}$ & $20 \pm 21$ & $35 \pm 26$ & $49 \pm 20$ & $47 \pm 20$ & $62 \pm 21$ & $71 \pm 25$ & $67 \pm 26$ \\
\hline & $m_{t>9 \mathrm{Gyr}}$ & $69 \pm 30$ & $55 \pm 28$ & $36 \pm 21$ & $40 \pm 21$ & $28 \pm 20$ & $19 \pm 24$ & $16 \pm 22$ \\
\hline \multirow{4}{*}{ M7 } & $m_{t<1 \mathrm{Gyr}}$ & $1 \pm 1$ & $1 \pm 1$ & $1 \pm 2$ & $1 \pm 4$ & $1 \pm 3$ & $2 \pm 4$ & $3 \pm 8$ \\
\hline & $m_{1<t<4 \mathrm{Gyr}}$ & $8 \pm 8$ & $5 \pm 9$ & $12 \pm 20$ & $8 \pm 15$ & $7 \pm 16$ & $6 \pm 14$ & $8 \pm 16$ \\
\hline & $m_{4<t<9 \mathrm{Gyr}}$ & $5 \pm 7$ & $4 \pm 6$ & $2 \pm 4$ & $4 \pm 6$ & $6 \pm 7$ & $10 \pm 10$ & $12 \pm 10$ \\
\hline & $m_{t>9 \mathrm{Gyr}}$ & $86 \pm 11$ & $90 \pm 10$ & $85 \pm 21$ & $87 \pm 19$ & $86 \pm 20$ & $82 \pm 21$ & $76 \pm 24$ \\
\hline \multirow{4}{*}{ M8 } & $m_{t<1 \mathrm{Gyr}}$ & $1 \pm 1$ & $2 \pm 6$ & $1 \pm 3$ & $1 \pm 3$ & $1 \pm 2$ & $2 \pm 3$ & $3 \pm 7$ \\
\hline & $m_{1<t<4 \mathrm{Gyr}}$ & $7 \pm 8$ & $3 \pm 7$ & $13 \pm 25$ & $9 \pm 20$ & $9 \pm 22$ & $12 \pm 25$ & $20 \pm 22$ \\
\hline & $m_{4<t<9 \mathrm{Gyr}}$ & $39 \pm 25$ & $36 \pm 27$ & $48 \pm 29$ & $50 \pm 23$ & $65 \pm 30$ & $70 \pm 25$ & $60 \pm 28$ \\
\hline & $m_{t>9 \mathrm{Gyr}}$ & $53 \pm 26$ & $59 \pm 28$ & $38 \pm 23$ & $39 \pm 25$ & $25 \pm 29$ & $16 \pm 21$ & $17 \pm 22$ \\
\hline \multirow{4}{*}{ M9 } & $m_{t<1 \mathrm{Gyr}}$ & $1 \pm 1$ & $1 \pm 1$ & $1 \pm 1$ & $2 \pm 1$ & $3 \pm 1$ & $4 \pm 1$ & $4 \pm 2$ \\
\hline & $m_{1<t<4 \mathrm{Gyr}}$ & $9 \pm 8$ & $8 \pm 7$ & $8 \pm 5$ & $9 \pm 4$ & $14 \pm 4$ & $16 \pm 4$ & $16 \pm 5$ \\
\hline & $m_{4<t<9 \mathrm{Gyr}}$ & $40 \pm 7$ & $39 \pm 7$ & $39 \pm 5$ & $39 \pm 4$ & $40 \pm 1$ & $40 \pm 1$ & $40 \pm 2$ \\
\hline & $m_{t>9 \mathrm{Gyr}}$ & $50 \pm 12$ & $52 \pm 11$ & $52 \pm 9$ & $50 \pm 7$ & $43 \pm 6$ & $40 \pm 5$ & $40 \pm 7$ \\
\hline
\end{tabular}


R. López Fernández et al.: Cosmic evolution of SFR, sSFR, and stellar mass density
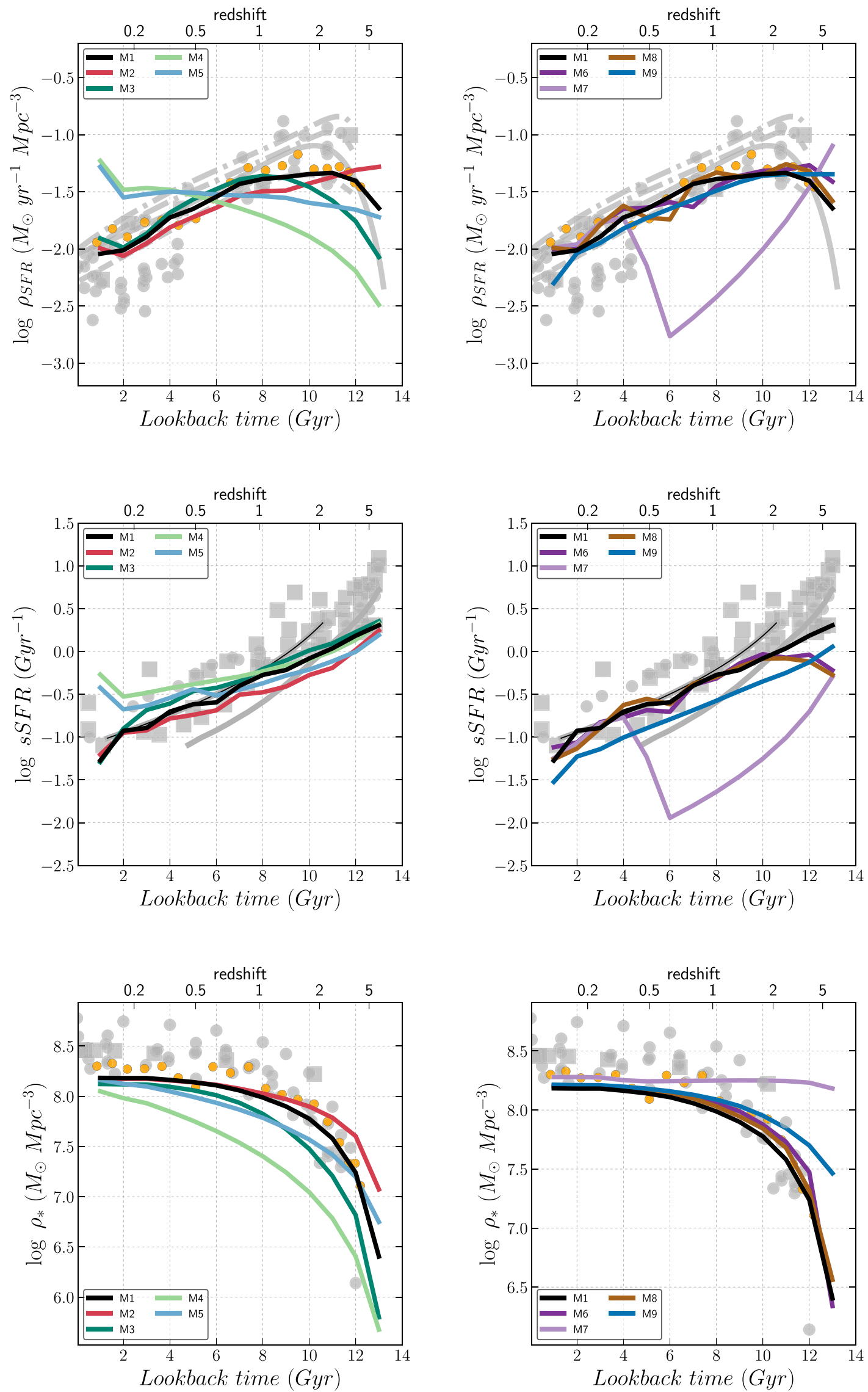

Fig. A.5. Cosmic evolution of SFR ( $\rho_{\mathrm{SFR}}$, upper panels), sSFR (middle panels), and stellar mass $\left(\rho_{\star}\right.$, bottom panels) for one single parametric SFH (left panels) and a combination of two parametric SFH (right panels). The reference delayed- $\tau$ model (M1, black line) is plotted in all the panels. Gray and yellow points and gray curves are taken from the literature as explained in the text and in the captions of Figs. 6-8. 